\title{
Spontaneous decay of artificial atoms in a three-qubit system
}

\author{
Ya. S. Greenberg* A. A. Shtygashev, and A. G. Moiseev \\ Novosibirsk State Technical University, Novosibirsk, Russia
}

(Dated: July 29, 2021)

\begin{abstract}
We study the evolution of qubits amplitudes in a one-dimensional chain consisting of three equidistantly spaced noninteracting qubits embedded in an open waveguide. The study is performed in the frame of single-excitation subspace, where the only qubit in the chain is initially excited. We show that the dynamics of qubits amplitudes crucially depend on the value of $k d$, where $k$ is the wave vector, $d$ is a distance between neighbor qubits. If $k d$ is equal to an integer multiple of $\pi$, then the qubits are excited to a stationary level. In this case, it is the dark states which prevent qubits from decaying to zero even though they do not contribute to the output spectrum of photon emission. For other values of $k d$ the excitations of qubits exhibit the damping oscillations which represent the vacuum Rabi oscillations in a three-qubit system. In this case, the output spectrum of photon radiation is determined by a subradiant state which has the lowest decay rate. We also investigated the case with the frequency of a central qubit being different from that of the edge qubits. In this case, the qibits decay rates can be controlled by the frequency detuning between the central and the edge qubits.
\end{abstract}

\section{INTRODUCTION}

Superconducting qubits coupled to photons propagating in an open waveguide [1 3] allow for the investigation of the fascinating world of quantum light-matter interactions in one dimension [4 6]. Even though the properties of multi-qubit $1 \mathrm{D}$ systems have been extensively studied both theoretically [7-11] and experimentally [12 14], less attention has been paid to a detail investigation of dynamic properties of the few-qubit systems which are the building blocks for quantum gates [15, 16]. Moreover, the scaling laws for decay rates that have been found for multi-qubit systems [17] cannot obviously be applied for systems containing few qubits.

As is known, the superconducting qubits can be technologically addressed and controlled individually [6]. Therefore, it is important to know the evolution of the probability amplitude of any qubit in a superconducting circuitry.

Here we explore the dynamic properties of a quantum circuit consisting of a three-qubit linear chain, which is strongly coupled to a common waveguide. The motivation for this choice is that it is a simplest system with non-trivial properties for which a full analytical treatment can be obtained. We investigate a dynamic behavior of the qubits amplitudes with the only qubit in the chain being initially excited. For three-qubit system, we find the analytic expressions for the complex energies and for the collective states which define a temporal behavior of qubits amplitudes. We show that the dynamics of qubits amplitudes crucially depend on the value of $k d$, where $k$ is the wave vector, $d$ is a distance between neighbor qubits. If $k d$ is equal to integer multiple of $\pi$, the qubits are excited to a stationary level. In this case, these are the dark states which prevent qubits from de-

*Electronic address: yakovgreenberg@yahoo.com caying to zero even though they do not contribute to the output photon spectrum. For other values of $k d$, the excitations of qubits have oscillatory behavior and are gradually damped out to zero. We also investigate the case with the frequency of a central qubit being different from that of the edge qubits. In this case, the qibits decay rates can be controlled by the frequency detuning between the central and the edge qubits. As the frequency detuning between central and edge qubits increases, the decay rates of qubits are also increases. This property is very important for the implementation of the efficient control and readout protocols where a fast reset of the excited qubits to their ground state is essential [18, 19].

The paper is structured as follows.

In Sec. II, we begin by introducing a JaynesCummings Hamiltonian for atom-light interactions. We truncate the Hilbert space to a single-excitation subspace and obtain a set of linear integro-differential equations for the qubits amplitudes.

In Sec. III, in the frame of Wigner-Weisskopf approximation we derive a set of linear differential equations for the qubits amplitudes $\beta_{n}(t)$, which allow for a direct numerical simulations.

A comprehensive analysis of the dynamics of the qubits amplitudes is given in Sec. IV. We show that for $k d=n \pi$ the qubits amplitudes become "frozen" at the constant level. The reason for this is the dark states which prevent qubits from decaying to zero. For the values of $k d$ which are not integer multiple of $\pi$ the qubits amplitudes gradually damp out to zero. In this section we also calculated the probability amplitude of the photon emission. We find, that for $k d=(2 n+1) \pi$ the evolution of the photon amplitude consists of clearly seen steps. These steps can be attributed to the interrelation between temporal behaviors of the different qubits amplitudes.

In Sec. $\mathrm{V}$ we calculate a spectral density of photon radiation from a three-qubit chain. We show that for $k d=n \pi$ the dark states do not contribute to output radiation near resonance. In this case, the output spectral 


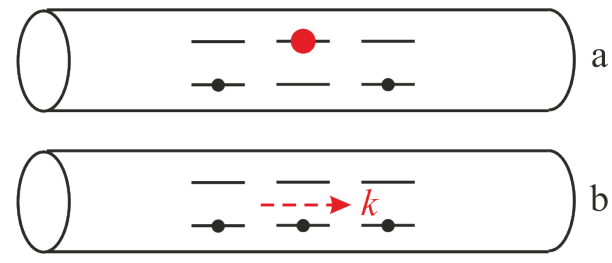

FIG. 1: Schematic illustration of a single-excitation subspace for a three-qubit chain in an open waveguide. (a) A single qubit is excited, two qubits are in the ground state. (b) three qubits are in the ground state and a single photon propagates in the waveguide.

density has a Lorentzian lineshape. For $k d=(2 n+1) \pi / 2$ a spectral density exhibits two peaks which are a signature of the vacuum Rabi oscillations of qubits amplitudes. In general, if $k d$ is not equal to an integer multiple of $\pi$, the width of a spectral line is defined by the deep subradiant states.

In Sec. VI we formulate the dynamics of a three-qubit chain with the aid of a non-Hermitian Hamiltonian which is obtained after the elimination of the photon variables. We find the collective states which are eigenvectors of non-Hermitian Hamiltonian and show how the qubits amplitudes $\beta_{n}(t)$ can be expressed in terms of these collective states.

In Sec. VII we consider a three-qubit system in which the frequency $\Omega_{0}$ of the central qubit is different from that of the edge qubits. We show that using the detuning $\delta \Omega=\Omega-\Omega_{0}$ as external parameter we can control the decay rates of the qubits' amplitudes. As the frequency detuning between central and edge qubits increases, the decay rates of qubits are also increases. For this case, a spectral density of photon radiation is also calculated for different values of $\delta \Omega$.

The main results of the paper are summarized in the concluding Section VIII.

\section{FORMULATION OF THE PROBLEM}

We consider a linear chain of three equally spaced qubits which are coupled to photon field in an open waveguide (see Fig. 1). A distance between neighbor qubits is equal to $d$. The Hilbert space of every qubit consists of the excited state $|e\rangle$ and the ground state $|g\rangle$. The Hamiltonian which accounts for the interaction between qubits and the electromagnetic field is as follows (we use $\hbar=1$ throughout the paper):

$$
H=H_{0}+\sum_{k} \omega_{k} a_{k}^{+} a_{k}+H_{\text {int }}
$$

where $H_{0}$ - is Hamiltonian of bare qubits.

$$
H_{0}=\frac{1}{2} \sum_{n=1}^{3}\left(1+\sigma_{z}^{(n)}\right) \Omega_{n}
$$

$$
H_{\mathrm{int}}=\sum_{n=1}^{3} \sum_{k} g_{k}^{(n)} e^{-i k x_{n}} \sigma_{-}^{(n)} a_{k}^{+}+\text {h.c. }
$$

The quantity $g_{k}^{(n)}$ in $(3)$ is the coupling between $n$-th qubit and the photon field in a waveguide. Below we consider a single-excitation subspace with either a single photon is in a waveguide and all qubits are in the ground state, Fig. 1 $\mathrm{b}$, or there are no photons in a waveguide with the only n-th qubit in the chain being excited, Fig. 17. Therefore, we truncate Hilbert space to the following states:

$$
\begin{aligned}
& \left|G, 1_{k}\right\rangle=\left|g_{1}, g_{2}, g_{3}\right\rangle \otimes\left|1_{k}\right\rangle ; \\
& \left|1,0_{k}\right\rangle=\left|e_{1}, g_{2}, g_{3}\right\rangle \otimes\left|0_{k}\right\rangle ; \\
& \left|2,0_{k}\right\rangle=\left|g_{1}, e_{2}, g_{3}\right\rangle \otimes\left|0_{k}\right\rangle ; \\
& \left|3,0_{k}\right\rangle=\left|g_{1}, g_{2}, e_{3}\right\rangle \otimes\left|0_{k}\right\rangle
\end{aligned}
$$

The Hamiltonian (3) preserves the number of excitations (number of excited qubits + number of photons). In our case the number of excitations is equal to one (see Fig. 1. Therefore, at any instant of time the system will remain within a single-excitation subspace. The wave function of an arbitrary single-excitation state can then be written in the form:

$$
|\Psi\rangle=\sum_{n=1}^{3} \beta_{n}(t) e^{-i \Omega_{n} t}\left|n, 0_{k}\right\rangle+\sum_{k} \gamma_{k}(t) e^{-i \omega_{k} t}\left|G, 1_{k}\right\rangle
$$

where $\beta_{n}(t)$ is the amplitude of $n$-th qubit, $\gamma_{k}(t)$ is a single-photon amplitude which is related to a spectral density of spontaneous emission.

$$
S\left(\omega_{k}, t\right)=\left|\gamma_{k}(t)\right|^{2} .
$$

The function (5) is normalized to unity:

$$
\sum_{n=1}^{3}\left|\beta_{n}(t)\right|^{2}+\sum_{k}\left|\gamma_{k}(t)\right|^{2}=1
$$

From (7) we can find the full probability of photon emission from the three-qubit system.

$$
P_{p h}(t)=\sum_{k}\left|\gamma_{k}(t)\right|^{2}=1-\sum_{n=1}^{3}\left|\beta_{n}(t)\right|^{2}
$$

In fact, the quantity $P_{p h}(t)$ is the probability to find the emitted photon at the moment $t$.

The equations for qubits amplitudes $\beta_{n}(t)$ in (5) can be found from time-dependent Schrodinger equation $i d|\Psi\rangle / d t=H|\Psi\rangle$. For the amplitudes $\gamma_{k}(t), \beta_{n}(t)$ we obtain: 


$$
\begin{gathered}
\frac{d \beta_{n}}{d t}=-\sum_{k}\left|g_{k}^{(n)}\right|^{2} \int_{0}^{t} \beta_{n}\left(t^{\prime}\right) e^{-i\left(\omega_{k}-\Omega_{n}\right)\left(t-t^{\prime}\right)} d t^{\prime} \\
-\sum_{m \neq n}^{3} \sum_{k} g_{k}^{*(n)} g_{k}^{(m)} e^{-i k\left(x_{m}-x_{n}\right)} e^{i\left(\Omega_{n}-\Omega_{m}\right) t} \\
\times \int_{0}^{t} \beta_{m}\left(t^{\prime}\right) e^{-i\left(\omega_{k}-\Omega_{m}\right)\left(t-t^{\prime}\right)} d t^{\prime} \\
\gamma_{k}(t)=-i \sum_{n=1}^{3} g_{k}^{(n)} e^{-i k x_{n}} \int_{0}^{t} \beta_{n}\left(t^{\prime}\right) e^{i\left(\omega_{k}-\Omega_{n}\right) t^{\prime}} d t^{\prime}
\end{gathered}
$$

According to 10 there are no photons in the system at $t=0$. Our goal is to find the evolution of the amplitudes $\beta_{n}(t)$ for any qubit in the chain when the only $n_{0}$-th qubit $\left(n_{0}=1,2\right.$, or 3$)$ is initially excited:

$$
\left\{\begin{array}{l}
\beta_{n_{0}}(0)=1 \\
\beta_{n}(0)=0, \quad n \neq n_{0}
\end{array}\right.
$$

\section{EQUATIONS FOR THE QUBITS AMPLITUDES}

We assume that the first and the third qubits are identical $\left(\Omega_{1}=\Omega_{3} \equiv \Omega, g_{k}^{(1)}=g_{k}^{(3)} \equiv g_{k}\right)$. The frequency and the coupling of the second qubit are different $\left(\Omega_{2} \equiv\right.$ $\left.\Omega_{0}, g_{k}^{(2)} \equiv g_{k}^{(0)}\right)$. A distance between central qubit and the edge qubits is equal to $d$. We take the origin in the location of the second qubit: $x_{1}=-d, x_{2}=0, x_{3}=+d$. In the frame of Wigner-Weisskopf approximation, the equations (9) for the qubits amplitudes can be reduced to the following set of linear differential equations (see Appendix A for the derivation).

$$
\begin{gathered}
\frac{d \bar{\beta}_{1}}{d t}=-\frac{\Gamma}{2} \bar{\beta}_{1}(t)-i \frac{\Omega-\Omega_{0}}{2} \bar{\beta}_{1}(t) \\
-\bar{\beta}_{2}(t) \frac{1}{2}\left(\frac{\Omega_{0}}{\Omega}\right)^{1 / 2} \sqrt{\Gamma \Gamma_{0}} e^{i k_{0} d}-\frac{\Gamma}{2} \bar{\beta}_{3}(t) e^{2 i k d} \\
\frac{d \bar{\beta}_{2}}{d t}=-\frac{\Gamma_{0}}{2} \bar{\beta}_{2}(t)+i \frac{\Omega-\Omega_{0}}{2} \bar{\beta}_{2}(t) \\
-\frac{1}{2}\left(\frac{\Omega}{\Omega_{0}}\right)^{1 / 2} \sqrt{\Gamma \Gamma_{0}} e^{i k d}\left(\bar{\beta}_{1}(t)+\bar{\beta}_{3}(t)\right) \\
\frac{d \bar{\beta}_{3}}{d t}=-\frac{\Gamma}{2} \bar{\beta}_{3}(t)-i \frac{\Omega-\Omega_{0}}{2} \bar{\beta}_{3}(t)-\bar{\beta}_{1}(t) \frac{\Gamma}{2} e^{2 i k d} \\
-\bar{\beta}_{2}(t) \frac{1}{2}\left(\frac{\Omega_{0}}{\Omega}\right)^{1 / 2} \sqrt{\Gamma \Gamma_{0}} e^{i k_{0} d}
\end{gathered}
$$

where

$$
\begin{cases}\bar{\beta}_{1,3}(t) & =e^{-i\left(\Omega-\Omega_{0}\right) t / 2} \beta_{1,3}(t), \\ \bar{\beta}_{2}(t) & =e^{i\left(\Omega-\Omega_{0}\right) t / 2} \beta_{2}(t)\end{cases}
$$

$k=\Omega / v_{g}, k_{0}=\Omega_{0} / v_{g}, \Gamma, \Gamma_{0}$ are the rates of spontaneous emission into the waveguide mode from edge qubits and from the central qubit, respectively.

\section{THE DYNAMICS OF THREE IDENTICAL QUBITS}

For three identical qubits we obtain from $(12)-(14)$ the following equations:

$$
\frac{d \beta_{n}}{d t}=-\frac{\Gamma}{2} \sum_{m=1}^{3} \beta_{m}(t) e^{i k d|m-n|}, \quad(n=1,2,3)
$$

which can be expanded explicitly as:

$$
\begin{aligned}
& \frac{d \beta_{1}}{d t}=-\frac{\Gamma}{2} \beta_{1}(t)-\frac{\Gamma}{2} \beta_{2}(t) e^{i k d}-\frac{\Gamma}{2} \beta_{3}(t) e^{2 i k d} \\
& \frac{d \beta_{2}}{d t}=-\frac{\Gamma}{2} \beta_{2}(t)-\frac{\Gamma}{2} e^{i k d}\left(\beta_{1}(t)+\beta_{3}(t)\right) \\
& \frac{d \beta_{3}}{d t}=-\frac{\Gamma}{2} \beta_{3}(t)-\frac{\Gamma}{2} \beta_{1}(t) e^{2 i k d}-\frac{\Gamma}{2} \beta_{2}(t) e^{i k d}
\end{aligned}
$$

where $k=\Omega / v_{g}$, and for simplicity we remove the bar over $\beta_{n}(t)$.

The general solution of equations (17) can be written as follows:

$$
\beta_{i}(t)=\sum_{m=1}^{3} a_{m}^{(i)} e^{\lambda_{m} t} ; i=1,2,3
$$

The quantities $\lambda_{m}$ are characteristic roots, which can be found by equating to zero the determinant of equations 17

$$
\left(\lambda_{i}+\frac{\Gamma}{2}\right) \delta_{m n}+\frac{\Gamma}{2} e^{i k d|m-n|}\left(1-\delta_{m n}\right)
$$

The quantities $a_{m}^{(n)}$ in $(18)$ are defined by initial conditions:

$$
\left\{\begin{array}{l}
\sum_{m=1}^{3} a_{m}^{\left(n_{0}\right)}=1, \\
\sum_{m=1}^{3} a_{m}^{(i)}=0, \quad i \neq n_{0} .
\end{array}\right.
$$

where $n_{0}$ is the number of initially excited qubit.

From determinant of 19 we obtain the exact expressions for $\lambda_{m}$ :

$$
\begin{gathered}
\lambda_{1}=-\frac{\Gamma}{4} e^{i 2 k d}-\frac{\Gamma}{4} e^{i k d} \sqrt{e^{i 2 k d}+8}-\frac{\Gamma}{2} \\
\lambda_{2}=-\frac{\Gamma}{4} e^{i 2 k d}+\frac{\Gamma}{4} e^{i k d} \sqrt{e^{i 2 k d}+8}-\frac{\Gamma}{2} \\
\lambda_{3}=\frac{\Gamma}{2} e^{i 2 k d}-\frac{\Gamma}{2}
\end{gathered}
$$

From 21 we see that the decay rates $\Gamma_{i}=-\operatorname{Re}\left(\lambda_{i}\right)$ depend on $k d$. This dependence is shown in Fig. 2

In general, as is seen from (21), all $\Gamma_{i}$ 's contribute to the decay rate of a concrete qubit. More important is that a sum

$$
\sum_{i=1}^{3} \lambda_{i}=-\frac{3}{2} \Gamma
$$




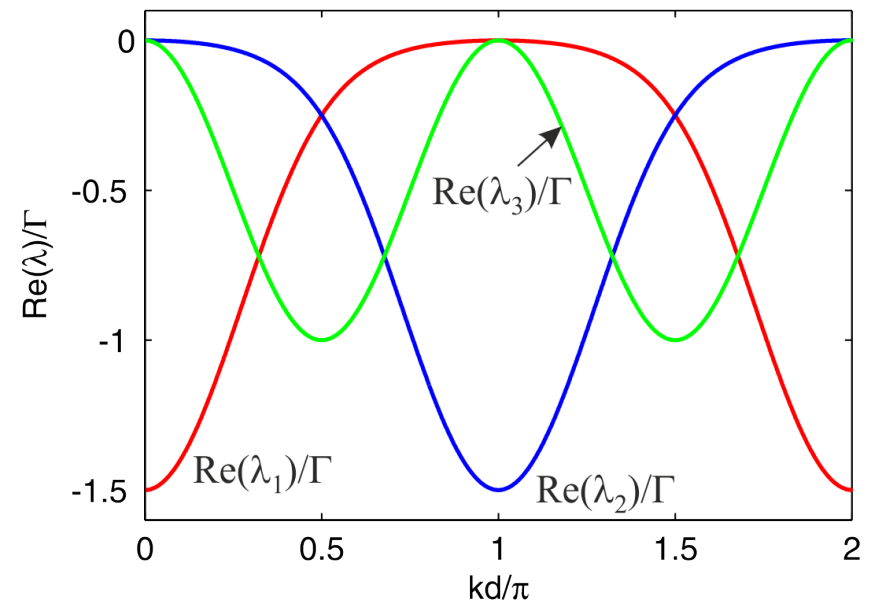

FIG. 2: Dependence of $\operatorname{Re}\left(\lambda_{i}\right)$ on $k d$. $\operatorname{Re}\left(\lambda_{1}\right)$-red line, $\operatorname{Re}\left(\lambda_{2}\right)$ blue line, $\operatorname{Re}\left(\lambda_{3}\right)$-green line. All values of $\operatorname{Re}\left(\lambda_{i}\right)$ are negative.

does not depend on $k d$. The expression 22 is a special case of the more general sum rule for $N$ qubit system.

$$
\sum_{i=1}^{N} \Gamma_{i}=N \Gamma
$$

where $\Gamma_{i}=2 \operatorname{Re}\left(\lambda_{i}\right)$. The sum rule 23 states that there are no other losses in the system other than the coherent spontaneous emission into a waveguide.

Consider now the solution of equations (17). By subtracting the third equation in (17) from the first one we obtain:

$$
\frac{d}{d t}\left(\beta_{1}-\beta_{3}\right)=-\frac{\Gamma}{2}\left(1-e^{i 2 k d}\right)\left(\beta_{1}-\beta_{3}\right)
$$

It follows from 24 that if the edge qubits are initially not excited, $\beta_{1}(0)-\beta_{3}(0)=0$, then this difference remains zero for all times, $\beta_{1}(t)=\beta_{3}(t) \equiv \beta(t)$. This is quite reasonable from symmetry consideration: if a central qubit is initially excited, the temporal behavior of the amplitudes of the edge qubits must be the same.

Therefore, for this case, three equations (17) can be reduced to two equations:

$$
\begin{aligned}
& \frac{d \beta_{2}}{d t}=-\frac{\Gamma}{2} \beta_{2}(t)-\Gamma \beta(t) e^{i k d} \\
& \frac{d \beta}{d t}=-\frac{\Gamma}{2} \beta(t)\left(1+e^{2 i k d}\right)-\frac{\Gamma}{2} \beta_{2}(t) e^{i k d}
\end{aligned}
$$

where $\beta_{2}(0)=1, \quad \beta(0)=0$.

The characteristic roots of (25) are equal to $\lambda_{1}$ and $\lambda_{2}$ which are given in 21. Therefore, the solution of equations 25 reads:

$$
\left\{\begin{array}{l}
\beta_{2}(t)=b_{1} e^{\lambda_{1} t}+b_{2} e^{\lambda_{2} t} \\
\beta(t)=a_{1} e^{\lambda_{1} t}+a_{2} e^{\lambda_{2} t}
\end{array}\right.
$$

where from initial conditions

$$
\left\{\begin{array}{l}
b_{1}+b_{2}=1 \\
a_{1}+a_{2}=0
\end{array}\right.
$$

Another two conditions follow from 25 for time derivatives at $t=0$.

$$
\left\{\begin{aligned}
b_{1} \lambda_{1}+b_{2} \lambda_{2} & =-\frac{\Gamma}{2} \\
a_{1} \lambda_{1}+a_{2} \lambda_{2} & =-\frac{\Gamma}{2} e^{i k d} .
\end{aligned}\right.
$$

From 27) and 28 we obtain

$$
\begin{gathered}
b_{1}=-\frac{\frac{\Gamma}{2}+\lambda_{2}}{\lambda_{1}-\lambda_{2}} ; b_{2}=\frac{\frac{\Gamma}{2}+\lambda_{1}}{\lambda_{1}-\lambda_{2}} \\
a_{1}=-\frac{\Gamma}{2} \frac{e^{i k d}}{\lambda_{1}-\lambda_{2}} ; a_{2}=\frac{\Gamma}{2} \frac{e^{i k d}}{\lambda_{1}-\lambda_{2}}
\end{gathered}
$$

Using the explicit expressions 21 we obtain

$$
\begin{aligned}
& a_{1}=\frac{1}{R} ; a_{2}=-\frac{1}{R} \\
& b_{1}=\frac{R-\mathrm{e}^{\mathrm{ikd}}}{2 R} ; b_{2}=\frac{R+e^{\mathrm{ikd}}}{2 R}
\end{aligned}
$$

where $R=\sqrt{e^{i 2 k d}+8}$.

Now we assume that the first qubit in the chain is initially excited.

$$
\beta_{1}(0)=1, \beta_{2}(0)=0, \beta_{3}(0)=0 \text {. }
$$

In this case, all amplitudes behave differently, so that the equations (17) should be used from which the solution can be straightforwardly obtained

$$
\begin{aligned}
& \beta_{1}(\mathrm{t})=\frac{b_{2}}{2} e^{\lambda_{1} t}+\frac{b_{1}}{2} e^{\lambda_{2} t}+\frac{1}{2} e^{\lambda_{3} t} \\
& \beta_{2}(\mathrm{t})=\frac{1}{R}\left(\mathrm{e}^{\lambda_{1} \mathrm{t}}-\mathrm{e}^{\lambda_{2} \mathrm{t}}\right) \\
& \beta_{3}(\mathrm{t})=\frac{b_{2}}{2} e^{\lambda_{1} t}+\frac{b_{1}}{2} e^{\lambda_{2} t}-\frac{1}{2} e^{\lambda_{3} t}
\end{aligned}
$$

where $b_{1}$ and $b_{2}$ are given in 31 . For special cases when $k d$ is integer multiple of $\pi$ we may obtain from (26) and (33) very simple forms for the temporal behavior of the qubits' amplitudes.

$$
\text { A. } k d=\pi n
$$

For this case, $\lambda_{1}=-\frac{3}{2} \Gamma, \lambda_{2}=\lambda_{3}=0$ if $n$ is even number, and $\lambda_{1}=0, \lambda_{2}=-\frac{3}{2} \Gamma, \lambda_{3}=0$ if $n$ is odd number. The calculations show that no matter which qibit 
in the chain is initially excited (central or edge qubit) its evolution is the same.

$$
\beta_{e x c}(t)=\frac{1}{3} e^{-3 \Gamma t / 2}+\frac{2}{3}
$$

The evolution of unexcited qubits are also the same within a phase factor of $\pi$.

$$
\beta_{\text {unexc }}(t)=(-1)^{n}\left(\frac{1}{3} e^{-3 \Gamma t / 2}-\frac{1}{3}\right)
$$

The expression (35) is valid for any $n$ if the central qubit is excited. If the first qubit is excited and $n$ is the odd number, the amplitudes of unexcited qubits evolve with the opposite phases: $\beta_{2}(t)=\beta_{\text {unexc }}(t), \beta_{3}(t)=$ $-\beta_{\text {unexc }}(t)$.

It worth noting the equality of the amplitudes of the second and the third qubit (if the first qubit is initially excited) even though they are located at different distance from the excited qubit. This is the consequence of Wigner-Weisskopf (or Markov) approximation: in this special case there are no interference between qubits so that all unexcited qubits feel the photon field simultaneously no matter how far they are from the excited qubit. From (34) and (35) we find a full probability of photon emission (8)

$$
P_{p h}(t)=1-2\left|\beta_{\text {unexc }}(t)\right|^{2}-\left|\beta_{\text {exc }}(t)\right|^{2}=\frac{1}{3}\left(1-e^{-3 \Gamma t}\right)
$$

The probability of qubits amplitudes and a full probability of photon emission are shown in Fig. 3

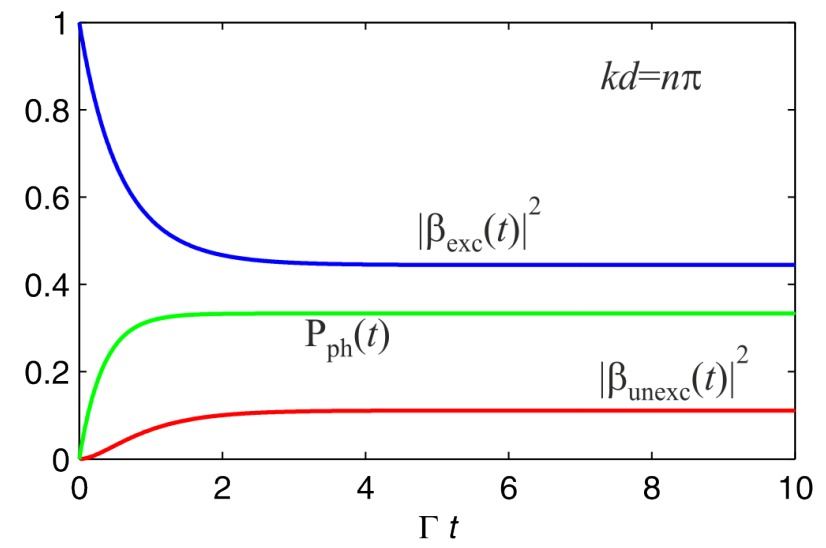

FIG. 3: The probability amplitudes for $k d=n \pi$ of excited, $\left|\beta_{\text {exc }}(t)\right|^{2}$ (the upper line) and unexcited $\left|\beta_{\text {unexc }}(t)\right|^{2}$ (the lower line) qubits. The probability of a full photon emission is shown between the qubit lines.

As is seen from Fig. 3, as the time proceeds the qubits amplitudes become "frozen" at some level. We see from (34) and (35) that this frozenness is solely due to the dark states $\left(\lambda_{n}=0\right)$, which prevent the qubits' amplitudes from decaying to zero. The frozenness can be lifted if $k d$ is not equal to an integer multiple of $\pi$. Then all qubits are damped to zero with the rate being determined by the root of $\lambda_{n}$ which has the lowest real part.

$$
\text { B. } k d=\pi(2 n+1) / 2
$$

If $\mathrm{n}$ is even number then $\lambda_{1}=-\frac{\Gamma}{4}(1+i \sqrt{7}), \lambda_{2}=$ $-\frac{\Gamma}{4}(1-i \sqrt{7}), \lambda_{3}=-\Gamma$, and if $\mathrm{n}$ is odd number then $\lambda_{1}=-\frac{\Gamma}{4}(1-i \sqrt{7}), \lambda_{2}=-\frac{\Gamma}{4}(1+i \sqrt{7}), \lambda_{3}=-\Gamma$

If the central qubit is initially excited we obtain:

$$
\begin{aligned}
& \beta_{1}(t)=\beta_{3}(t)=(-1)^{n+1} i \frac{2}{\sqrt{7}} e^{-\frac{1}{4} \Gamma t} \sin \left(\frac{\sqrt{7}}{4} \Gamma t\right) \\
& \beta_{2}(t)=e^{-\frac{1}{4} \Gamma t}\left(\cos \left(\frac{\sqrt{7}}{4} \Gamma t\right)+(-1)^{n} \frac{1}{\sqrt{7}} \sin \left(\frac{\sqrt{7}}{4} \Gamma t\right)\right)
\end{aligned}
$$

For initially excited edge qubit we obtain:

$$
\begin{aligned}
& \beta_{1}(t)=\frac{e^{-\frac{1}{4} \Gamma t}}{2}\left(\cos \left(\frac{\sqrt{7}}{4} \Gamma t\right)+\frac{1}{\sqrt{7}} \sin \left(\frac{\sqrt{7}}{4} \Gamma t\right)\right)+\frac{1}{2} e^{-\Gamma t} \\
& \beta_{2}(t)=(-1)^{n+1} i \frac{2}{\sqrt{7}} e^{-\frac{1}{4} \Gamma t} \sin \left(\frac{\sqrt{7}}{4} \Gamma t\right) \\
& \beta_{3}(t)=\frac{e^{-\frac{1}{4} \Gamma t}}{2}\left(\cos \left(\frac{\sqrt{7}}{4} \Gamma t\right)+\frac{1}{\sqrt{7}} \sin \left(\frac{\sqrt{7}}{4} \Gamma t\right)\right)-\frac{1}{2} e^{-\Gamma t}
\end{aligned}
$$

The oscillatory behavior of the qubits amplitudes in these expressions is a signature of the vacuum Rabi oscillations with the frequency $\sqrt{7} \Gamma / 4$.

The evolution of qubits amplitudes for half-integer multiple of $\pi$ with the central qubit being initially excited is shown in Fig. 4 .

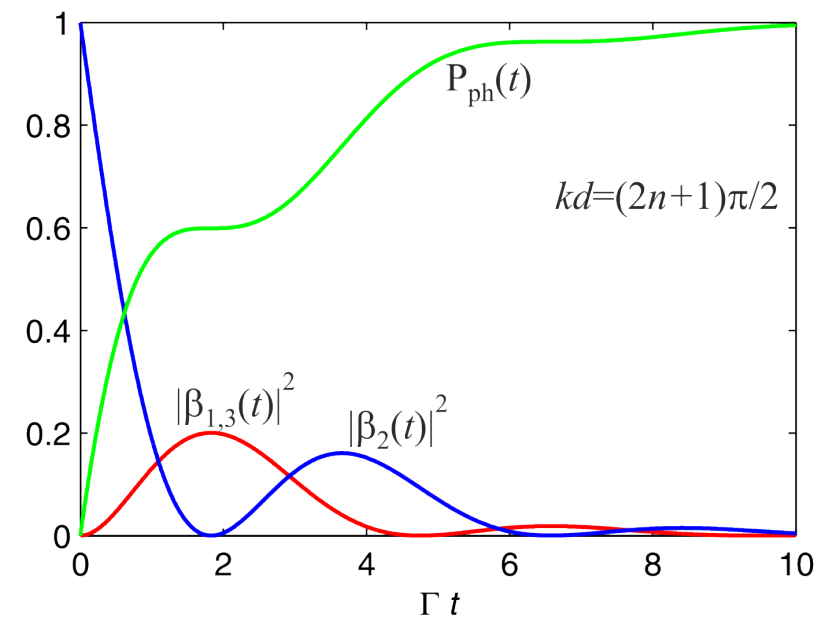

FIG. 4: The evolution of qubits amplitudes for $k d$ equals the half-integer multiple of $\pi$ with the central qubit being initially excited.

The evolution of $P_{p h}(t)$ reveals two clear visible steps where, $d P_{p h}(t) / d t=0$. These steps can be attributed 
to the interrelation between the temporal dynamics of the different amplitudes. As is seen from this figure, the steps on $P_{p h}(t)$ curve are in the vicinity of extremum points of qubits amplitudes. Physically, these steps are the signature of the trapping of the photon radiation. As is seen in Fig. 3, near the first step the radiation emitted by excited qubit is absorbed by unexcited qubits, so that the rate of the output radiation is not changed. The evolution of qubits' amplitudes for half-integer multiple of $\pi$ with the first qubit being initially excited is shown in Fig. 5 .

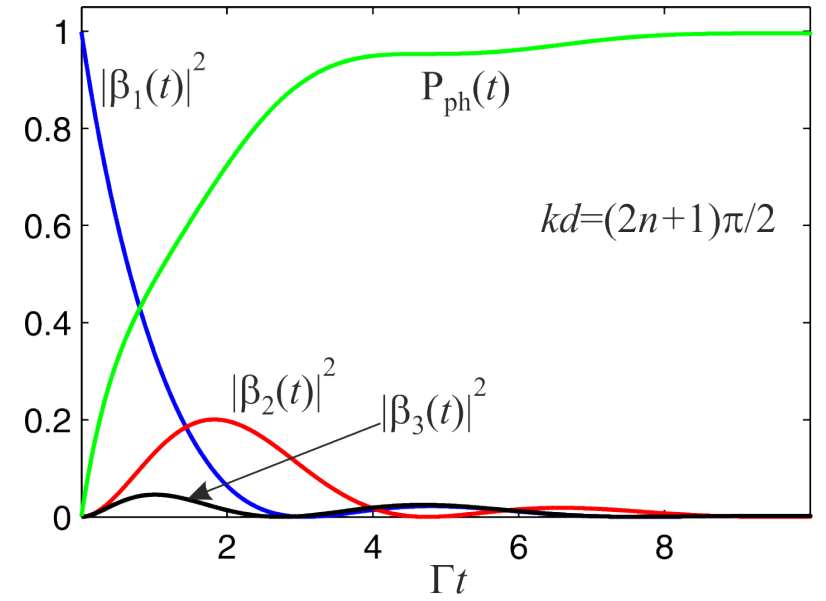

FIG. 5: The evolution of qubits amplitudes for $k d$ equals the half-integer multiple of $\pi$ with the first qubit being initially excited

Here, we see that the evolution of qubits amplitude depends on their distance from the excited qubit. The greater is the distance of a qubit from the excited one, the less it is affected by the excitation.

\section{SPECTRAL DENSITY OF PHOTON RADIATION}

The quantity $\gamma_{k}(t)$ in (5) allows for the calculation of a spectral density (6) of spontaneous emission into a waveguide, $S(\omega, t)$. The equation 10 for identical qubits reads:

$$
\gamma(\omega, t)=-i g_{k} \sum_{n=1}^{3} e^{-i k x_{n}} \int_{0}^{t} \beta_{n}\left(t^{\prime}\right) e^{i(\omega-\Omega) t^{\prime}} d t^{\prime}
$$

Using the expression 18 for $\beta_{n}(t)$ we obtain

$$
\gamma(\omega, t)=g_{k} \sum_{n, m=1}^{3} a_{j}^{(n)} e^{-i k x_{n}} \frac{1-e^{i\left(\omega-\Omega-i \lambda_{m}\right) t}}{\omega-\Omega-i \lambda_{m}}
$$

As $g_{k}$ in (39) and (40) depends on the waveguide length, $L$ and the light velocity $v_{g}$ (see (A16)), we factorize $\gamma_{k}(t)$ as follows:

$$
\gamma_{k}(t) \equiv\left(\frac{v_{g}}{2 L \Omega}\right)^{1 / 2}\left(\frac{\Gamma}{\Omega}\right)^{1 / 2} f_{k}(t)
$$

Therefore, we define spectral density in the following form:

$$
S(\omega, t)=\frac{\left|\gamma_{k}(t)\right|^{2}}{\left(\frac{v_{g}}{2 L \Omega}\right)}=\frac{\Gamma}{\Omega}\left|f_{k}(t)\right|^{2}
$$

The equation 40 is a rather general expression. As the time proceeds only the term with the lowest real part of $\lambda_{m}$ survives. The concise analytical results can be obtained only for several simple cases. Below we calculate the spectral density for $k d=n \pi$, using the amplitudes (34) and (35) in (39).

When a central qubit is initially excited we obtain for $\gamma_{k}(t)$ the following result:

$$
\begin{aligned}
& \gamma_{k}(t)=-g_{k} \frac{2}{3}\left(1-(-1)^{n} \cos \left(\frac{\omega}{\Omega} n \pi\right)\right) \frac{e^{i(\omega-\Omega) t}-1}{\omega-\Omega} \\
& -g_{k} \frac{1}{3}\left(1+(-1)^{n} 2 \cos \left(\frac{\omega}{\Omega} n \pi\right)\right) \frac{e^{i\left(\omega-\Omega+i \frac{3 \Gamma}{2}\right) t}-1}{\left(\omega-\Omega+i \frac{3 \Gamma}{2}\right)}
\end{aligned}
$$

If the first qubit is initially excited we obtain:

$$
\begin{aligned}
& \gamma_{k}(t)=-g_{k} \frac{1}{3}\left(2 e^{i\left(\frac{\omega}{\Omega} n \pi\right)}-e^{-i\left(\frac{\omega}{\Omega} n \pi\right)}-(-1)^{n}\right) \frac{e^{i(\omega-\Omega) t}-1}{\omega-\Omega} \\
& -g_{k} \frac{1}{3}\left((-1)^{n}+2 \cos \left(\frac{\omega}{\Omega} n \pi\right)\right) \frac{e^{i\left(\omega-\Omega+i \frac{3 \Gamma}{2}\right) t}-1}{\left(\omega-\Omega+i \frac{3 \Gamma}{2}\right)}
\end{aligned}
$$

It worth noting that in expressions $(42),(43)$ a wave vector $k$ is related to a running frequency $\omega: k=\omega / v_{g}$. The first lines in 42 and (43) are related to the dark states $\left(\lambda_{n}=0\right)$. They do not lead to a singularity near resonance $\omega \approx \Omega$, since $k d$ - dependent prefactors in this terms tend to zero more rapidly than the denominator does. At the point of a resonance these terms exactly equal to zero. A physical reason for this is that the dark states do not interact with a photon field and, therefore, cannot contribute to the photon emission. Therefore, near the resonance, a spectral density of spontaneous emission can be approximated by a Lorentzian form with a full width at half the height of the resonance line being equal to $3 \Gamma$.

$$
\begin{gathered}
\gamma_{k}(t) \approx g_{k} \frac{e^{i\left(\omega-\Omega+i \frac{3 \Gamma}{2}\right) t}-1}{\left(\omega-\Omega+i \frac{3 \Gamma}{2}\right)} \underset{t \rightarrow \infty}{\longrightarrow} \frac{g_{k}}{\left(\omega-\Omega+i \frac{3 \Gamma}{2}\right)} \\
S(\omega, t \rightarrow \infty)=\frac{\Gamma \Omega}{(\omega-\Omega)^{2}+\left(\frac{3 \Gamma}{2}\right)^{2}}
\end{gathered}
$$

For $k d=(2 n+1) \pi / 2$, the qubits amplitudes are given in equations (37) and (38). The calculation of (39) with 
these amplitudes results in the following expressions for $\gamma_{k}(t)$ with the central qubit being initially excited:

$$
\begin{aligned}
& \gamma_{k}(t)=-g_{k}\left[\frac{1}{2}\left(1-\frac{(-1)^{n} i}{\sqrt{7}}\right)-\frac{2(-1)^{n}}{\sqrt{7}} \cos k d\right] \\
& \times \frac{e^{i\left(\omega-\Omega+\frac{\sqrt{7}}{4} \Gamma+i \frac{1}{4} \Gamma\right) t}-1}{\omega-\Omega+\frac{\sqrt{7}}{4} \Gamma+i \frac{1}{4} \Gamma} \\
& -g_{k}\left[\frac{1}{2}\left(1+\frac{(-1)^{n} i}{\sqrt{7}}\right)+\frac{2(-1)^{n}}{\sqrt{7}} \cos k d\right] \\
& \times \frac{e^{i\left(\omega-\Omega-\frac{\sqrt{7}}{4} \Gamma+i \frac{1}{4} \Gamma\right) t}-1}{\omega-\Omega-\frac{\sqrt{7}}{4} \Gamma+i \frac{1}{4} \Gamma}
\end{aligned}
$$

If the edge qubit is initially excited we obtain:

$$
\begin{aligned}
& \gamma_{k}(t)=-i g_{k}\left[\frac{1}{2}\left(1-\frac{i}{\sqrt{7}}\right) \cos k d+\frac{(-1)^{n}}{\sqrt{7}}\right] \\
& \times \frac{e^{i\left(\omega-\Omega+\frac{\sqrt{7}}{4} \Gamma+i \frac{1}{4} \Gamma\right) t}-1}{\omega-\Omega+\frac{\sqrt{7}}{4} \Gamma+i \frac{1}{4} \Gamma} \\
& -i g_{k}\left[\frac{1}{2}\left(1+\frac{i}{\sqrt{7}}\right) \cos k d-\frac{(-1)^{n}}{\sqrt{7}}\right] \\
& \times \frac{e^{i\left(\omega-\Omega-\frac{\sqrt{7}}{4} \Gamma+i \frac{1}{4} \Gamma\right) t}-1}{\omega-\Omega-\frac{\sqrt{7}}{4} \Gamma+i \frac{1}{4} \Gamma} \\
& -i g_{k} \sin k d \frac{e^{i\left(\omega-\Omega+i \frac{1}{2} \Gamma\right) t}-1}{\omega-\Omega+i \frac{1}{2} \Gamma}
\end{aligned}
$$

In expressions 46, 47) $k d=\frac{\omega}{\Omega}(2 n+1) \pi / 2$.

The spectral density of photon radiation (41) calculated from (46) and 47) for $n=1, k d=1.5 \pi, t=$ $20 / \Gamma, \Gamma / \Omega=10^{-3}$ is shon in Fig. 6. Two peaks at these plots are a clear signature of vacuum Rabi oscillations..

\section{EFFECTIVE NON-HERMITIAN HAMILTONIAN AND COLLECTIVE STATES}

The elimination of photon variables allows us to express the photon-mediated interaction between identical qubits in terms of a non-Hermitian effective Hamiltonian, which in the Markovian approximation reads [11, 20]:

$$
H_{e f f}=-i \frac{\Gamma}{2} \sum_{m, n=1}^{3} e^{i k\left|x_{m}-x_{n}\right|} \sigma_{m}^{+} \sigma_{n}
$$

where $k=\Omega / v_{g}, v_{g}$ is the velocity of electromagnetic wave in a waveguide, $x_{n}$ is the position of $n$-th qubit, $\sigma_{n}^{+}, \sigma_{n}$ are raising and lowering spin operators for $n$-th qubit.

The rate of spontaneous emission $\Gamma$ of an individual qubit is defined by the Fermi golden rule:

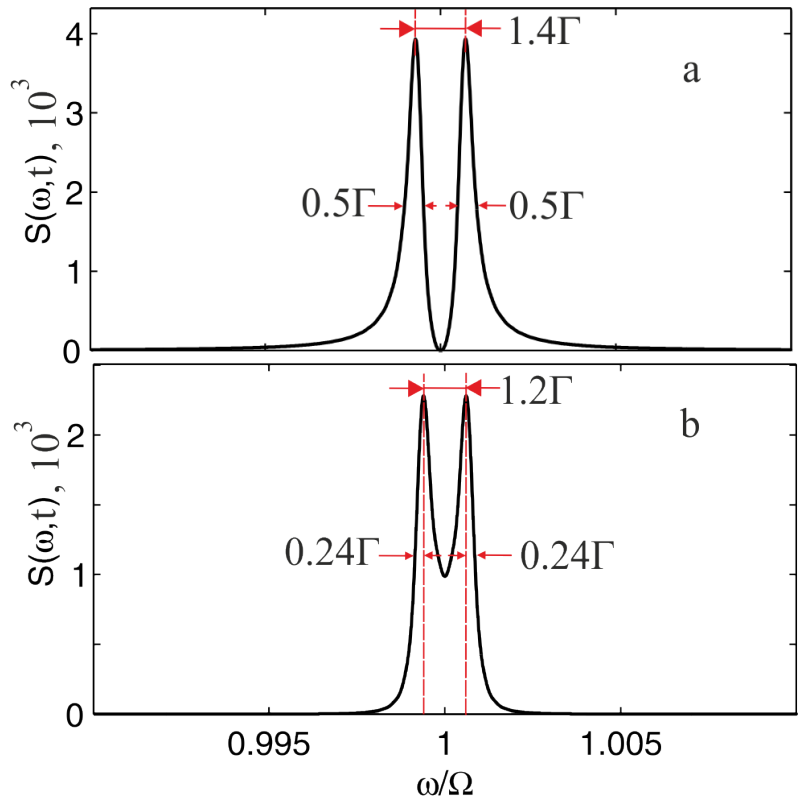

FIG. 6: Spectral density for three-qubit system calculated from (46) and (47) for $n=1, k d=1.5 \pi, t=20 / \Gamma, \Gamma / \Omega=$ $10^{-3}$. (a) second qubit is initially excited, (b) first qubit is initially excited.

$$
\Gamma=2 \pi \sum_{k}\left|g_{k}\right|^{2} \delta\left(\omega_{k}-\Omega\right)
$$

It follows from 48 that the photon-mediated interaction between qubits in such a system results in the coherent $J_{m n}=\Gamma \sin \left(k\left|x_{m}-x_{n}\right|\right) / 2$ and dissipative $\Gamma_{m n}=\Gamma \cos \left(k\left|x_{m}-x_{n}\right|\right)$ rates. The coherent rate shifts the positions of the qubits resonances, while the dissipative rate gives rise to the additional spontaneous emission into the waveguide mode. Unlike the real atoms with short-range dipole-dipole interaction, here a coherent interaction $J_{m n}$ is a long-range one: every qubit is sensitive to its distant neighbor.

The wave function for Hamiltonian 48 can be expressed in terms of a superposition of the single excited states

$$
\Psi(t)=\sum_{n=1}^{3} \beta_{n}(t)|n\rangle
$$

Even though the wave functions (5) and 50 are different, the qubits amplitudes $\beta_{n}(t)$ in these expressions are the same quantities'. Indeed, the equations for $\beta_{n}(t)$ in (50) can be derived from the time-dependent Schrodinger equation $i d \Psi / d t=H_{e f f} \Psi$ :

$$
\frac{d \beta_{n}}{d t}=-\frac{\Gamma}{2} \sum_{m=1}^{3} \beta_{m}(t) e^{i k\left|x_{m}-x_{n}\right|}, \quad(n=1,2,3)
$$


The equations (51) are nothing but a set of equations (17). Therefore, within a single excitation subspace two Hamiltonians, (1) and (48) are equivalent in that they provide the same equations for the qubits amplitudes.

In a single-excitation subspace, the Hamiltonian 48 has three collective eigenfunctions:

$$
\left|\Psi_{i}(t)\right\rangle=e^{-i \bar{E}_{i} t} \sum_{n=1}^{3} \alpha_{n}^{(i)}|n\rangle ;(i=1,2,3)
$$

where $\bar{E}_{i}$ is a complex energy

$$
\bar{E}_{i}=E_{i}-i \frac{\Gamma_{i}}{2}
$$

The quantities $E_{i}$ and $\Gamma_{i}$ depend on the system parameters $\Gamma, k, x_{n}$. The wave vectors with $\Gamma_{i}<\Gamma$ are called subradiant states, those with $\Gamma_{i}>\Gamma$ are called superradiant states. The coefficients in 52 can be obtained from the Schrodinger equation $H_{\text {eff }} \Psi=E \Psi$.

$E_{j} \sum_{n=1}^{3} \alpha_{n}^{(j)}|n\rangle=-i \frac{\Gamma}{2} \sum_{m, n=1}^{3} e^{i k d|m-n|} \alpha_{n}^{(j)}|m\rangle ; j=1,2,3$

Complex energies can be found by equating to zero the determinant of the matrix:

$$
\left(E+i \frac{\Gamma}{2}\right) \delta_{m n}+i \frac{\Gamma}{2} e^{i k d|m-n|}\left(1-\delta_{m n}\right) ; \quad(m, n=1,2,3)
$$

A comparison between the determinants of $(19)$ and (55) shows that the quantities $\lambda_{n}$ are related to those of complex energies, $\bar{E}_{n}: \bar{E}_{n}=i \lambda_{n}$ where $\lambda_{n}$ are given in (21).

From (54) we obtain three equations for the coefficients, .

$$
\begin{aligned}
& \left(E_{j}+i \frac{\Gamma}{2}\right) \alpha_{1}^{(j)}+i \frac{\Gamma}{2} e^{i k d} \alpha_{2}^{(j)}+i \frac{\Gamma}{2} e^{2 i k d} \alpha_{3}^{(j)}=0 \\
& \left(E_{j}+i \frac{\Gamma}{2}\right) \alpha_{2}^{(j)}+i \frac{\Gamma}{2} e^{i k d} \alpha_{1}^{(j)}+i \frac{\Gamma}{2} e^{i k d} \alpha_{3}^{(j)}=0 \\
& \left(E_{j}+i \frac{\Gamma}{2}\right) \alpha_{3}^{(j)}+i \frac{\Gamma}{2} e^{i k d} \alpha_{2}^{(j)}+i \frac{\Gamma}{2} e^{2 i k d} \alpha_{1}^{(j)}=0
\end{aligned}
$$

where for simplicity we remove the bar over $E_{i}$.

Because Hamiltonian (48) is non-Hermitian, the eigenfunctions 52 are neither normalized nor orthonormal. It is known that a correct calculation of the coefficients $\alpha_{n}^{(i)}$ in 52 requires a bi-orthogonal set of eigenfunctions $\left|\bar{\Psi}_{i}(t)\right\rangle$ which are a solution of the Schrodinger equation for $H_{e f f}^{\dagger}$. In our case $H_{e f f}^{\dagger}=H_{e f f}^{*}$ with the consequence that the complex conjugate of an eigenstate $\left|\Psi_{i}(t)\right\rangle$ of $H_{\text {eff }}$ is an eigenstate of $H_{\text {eff }}^{\dagger}$. Therefore, the conditions for normalization and orthonormality between eigenfunctions of these two sets lead to the following equations for the coefficients [21, 22].

$$
\begin{gathered}
\sum_{n=1}^{3}\left(\alpha_{n}^{(i)}\right)^{2}=1, \quad i=1,2,3 \\
\sum_{n=1}^{3} \alpha_{n}^{(i)} \alpha_{n}^{(j)}=0, \quad i \neq j, \quad i, j=1,2,3
\end{gathered}
$$

It worth noting that the coefficients $\alpha_{n}^{(i)}$ in (57) and (58) are in general complex quantities. In what follows we use equations (56) and the conditions (57) and (58) for the calculation of the coefficients $\alpha_{n}^{(i)}$.

As the energies $E_{j}$ are obtained from the determinant of equations (56), these equations are not independent. Therefore, for the calculations of the coefficients $\alpha_{n}^{(i)}$ we may take any two of them. For subsequent calculations we take first and second equations in (56). First, we calculate the coefficients $\alpha_{n}^{(1)}$. With $E_{1}=i \lambda_{1}$ determined in (21) two first equations in 56 read as follows:

$$
\begin{aligned}
& \left(e^{i k d}+R\right) \alpha_{1}^{(1)}-2 \alpha_{2}^{(1)}-2 e^{i k d} \alpha_{3}^{(1)}=0 \\
& \left(e^{i k d}+R\right) \alpha_{2}^{(1)}-2 \alpha_{1}^{(1)}-2 \alpha_{3}^{(1)}=0
\end{aligned}
$$

where $R$ is given in (31).

Additional third equation is given by the normalizing condition (57). Therefore, for the wave-function $\left|\Psi_{1}(t)\right\rangle$ we find the following set of the coefficients $\alpha_{n}^{(1)}$.

$$
\begin{aligned}
\alpha_{1}^{(1)}=\alpha_{3}^{(1)} & = \pm \frac{e^{i k d}\left(e^{i k d}+R\right)+2}{D(R)} \\
\alpha_{2}^{(1)} & = \pm \frac{3 e^{i k d}+R}{D(R)}
\end{aligned}
$$

where

$$
D(R)=\left(4 e^{4 i k d}+34 e^{2 i k d}+14 e^{i k d} R+4 e^{3 i k d} R+16\right)^{1 / 2}
$$

Similar calculations for $E_{2}=i \lambda_{2}$ provide the result that differs from (60), (61) only by the sign of $R$. Therefore, for the wave-function $\left|\Psi_{2}(t)\right\rangle$ we find the following set of the coefficients $\alpha_{n}^{(2)}$.

$$
\begin{aligned}
\alpha_{1}^{(2)}=\alpha_{3}^{(2)} & = \pm \frac{e^{i k d}\left(e^{i k d}-R\right)+2}{D(-R)} \\
\alpha_{2}^{(2)} & = \pm \frac{3 e^{i k d}-R}{D(-R)}
\end{aligned}
$$


The signs in right hand side of equations 60, 61, and (63), (64) must be the same (plus or minus) for all four expressions. This requirement follows from the orthonormality condition (58):

$$
\alpha_{1}^{(1)} \alpha_{1}^{(2)}+\alpha_{2}^{(1)} \alpha_{2}^{(2)}+\alpha_{3}^{(1)} \alpha_{3}^{(2)}=0
$$

Finally, for $E_{3}=i \lambda_{3}$ we obtain two equations

$$
\begin{aligned}
& \alpha_{1}^{(3)}+e^{-i k d} \alpha_{2}^{(3)}+\alpha_{3}^{(3)}=0 \\
& \alpha_{1}^{(3)}+e^{i k d} \alpha_{2}^{(3)}+\alpha_{3}^{(3)}=0
\end{aligned}
$$

which provide with account for the normalizing condition (57) the following result:

$$
\alpha_{1}^{(3)}= \pm \frac{1}{\sqrt{2}} ; \alpha_{3}^{(3)}=\mp \frac{1}{\sqrt{2}} ; \alpha_{2}^{(3)}=0 ;
$$

Therefore, the coefficients of collective states $\left|\Psi_{1}(t)\right\rangle$ and $\left|\Psi_{2}(t)\right\rangle$ depend on $k d$, while those of $\left|\Psi_{3}(t)\right\rangle$ are $k d$ independent.

Below, we consider special cases. For $k d=2 \pi n$, where $n$ is integer, we obtain from 60, 61) $\alpha_{1}^{(1)}=\alpha_{2}^{(1)}=$ $\alpha_{3}^{(1)}= \pm \frac{1}{\sqrt{3}}$. However, the calculation of the coefficients $\alpha_{n}^{(2)}$ from 63, 64 is not straightforward: at this point both the numerator and the denominator in these equations are equal to zero. In order to resolve this uncertainty we put in these equations $k d=2 \pi n+\epsilon$, where $\epsilon$ tends to zero. In this case, both the numerator and the denominator tend to zero as $\epsilon$, and their ratio is finite. The calculations show that as $k d$ tends to $2 \pi n$ the coefficients $\alpha_{n}^{(2)}$ tend to their finite values: $\alpha_{1}^{(2)}=\alpha_{3}^{(2)}=\frac{1}{\sqrt{6}} ; \alpha_{2}^{(2)}=-\frac{2}{\sqrt{6}}$.
For $k d=(2 n+1) \pi$ the picture is vise versa: $\alpha_{1}^{(1)}=$ $\alpha_{3}^{(1)}=\frac{1}{\sqrt{6}} ; \quad \alpha_{2}^{(1)}=-\frac{2}{\sqrt{6}}$, while $\alpha_{1}^{(2)}=\alpha_{2}^{(2)}=\alpha_{3}^{(2)}=$ $\pm \frac{1}{\sqrt{3}}$.

For $k d=(2 n+1) \pi / 2$ we obtain from (60), 61 , and 63), 64):

$$
\begin{aligned}
& \alpha_{1}^{(1)}=\alpha_{3}^{(1)}=\frac{-\mathrm{i}+(-1)^{n} \sqrt{7}}{\sqrt{2}\left(7-\mathrm{i}(-1)^{n} 5 \sqrt{7}\right)^{1 / 2}} \\
& \alpha_{2}^{(1)}=\frac{(-1)^{n} 3-\mathrm{i} \sqrt{7}}{\sqrt{2}\left(7-\mathrm{i}(-1)^{n} 5 \sqrt{7}\right)^{1 / 2}}
\end{aligned}
$$

$$
\begin{aligned}
& \alpha_{1}^{(2)}=\alpha_{3}^{(2)}=\frac{-\mathrm{i}-(-1)^{n} \sqrt{7}}{\sqrt{2}\left(7+i(-1)^{n} 5 \sqrt{7}\right)^{1 / 2}} \\
& \alpha_{2}^{(2)}=\frac{(-1)^{n} 3+i \sqrt{7}}{\sqrt{2}\left(7+i(-1)^{n} 5 \sqrt{7}\right)^{1 / 2}}
\end{aligned}
$$

Below we summarize these results in the explicit forms of collective wave functions for above special cases. If $k d$ is equal to integer multiple of $\pi$ we obtain:

$$
\begin{aligned}
& \left|\Psi_{1^{\natural}}(t)\right\rangle=e^{-\frac{3}{2} \Gamma t} \frac{1}{\sqrt{3}}(|1\rangle+|2\rangle+|3\rangle) \\
& \left|\Psi_{2^{\natural}}(t)\right\rangle=\frac{1}{\sqrt{6}}(|1\rangle-2|2\rangle+|3\rangle) \\
& \left|\Psi_{3^{\natural}}(t)\right\rangle=\frac{1}{\sqrt{2}}(|1\rangle-|3\rangle)
\end{aligned}
$$

Therefore, two of these collective states $\left|\Psi_{2}(t)\right\rangle$ and $\left|\Psi_{3}(t)\right\rangle$ are dark as their decay widths are zero.

For $k d=(2 n+1) \pi / 2$ the collective wave functions are as follows:

$$
\begin{aligned}
\left|\Psi_{1^{\prime}}(t)\right\rangle & =\frac{e^{-\frac{1}{4} \Gamma t} e^{-i \frac{\sqrt{7}}{4}(-1)^{n} \Gamma t}}{\sqrt{2}\left(7-i(-1)^{n} 5 \sqrt{7}\right)^{1 / 2}}\left(\left(-i+(-1)^{n} \sqrt{7}\right)(|1\rangle+|3\rangle)+\left((-1)^{n} 3-i \sqrt{7}\right)|2\rangle\right) \\
\left|\Psi_{2^{\prime}}(t)\right\rangle & =-\frac{e^{-\frac{1}{4} \Gamma t} e^{i \frac{\sqrt{7}}{4}(-1)^{n} \Gamma t}}{\sqrt{2}\left(7+i(-1)^{n} 5 \sqrt{7}\right)^{1 / 2}}\left(\left(i+(-1)^{n} \sqrt{7}\right)(|1\rangle+|3\rangle)-\left((-1)^{n} 3+i \sqrt{7}\right)|2\rangle\right) \\
\left|\Psi_{3^{\prime}}(t)\right\rangle & =e^{-\Gamma t} \frac{1}{\sqrt{2}}(|1\rangle-|3\rangle)
\end{aligned}
$$

where $n$ is any integer.

It is instructive to rewrite (71) in terms of the dark, $|D\rangle$ and bright, $|B\rangle$ states for a two qubits (the first qubit and the third one) separated by $\lambda / 2$. For $n=0$ we obtain from (71): 


$$
\begin{aligned}
& \left|\Psi_{1^{\prime}}(t)\right\rangle=\frac{e^{-\frac{1}{4} \Gamma t} e^{-i \frac{\sqrt{7}}{4} \Gamma t}}{\sqrt{2}(7-i 5 \sqrt{7})^{1 / 2}}\left((-i+\sqrt{7}) \sqrt{2}|D\rangle \otimes\left|g_{2}\right\rangle+(3-i \sqrt{7})|G\rangle \otimes\left|e_{2}\right\rangle\right) \\
& \left|\Psi_{2^{\prime}}(t)\right\rangle=-\frac{e^{-\frac{1}{4} \Gamma t} e^{i \frac{\sqrt{7}}{4} \Gamma t}}{\sqrt{2}(7+i 5 \sqrt{7})^{1 / 2}}\left((i+\sqrt{7}) \sqrt{2}|D\rangle \otimes\left|g_{2}\right\rangle-(3+i \sqrt{7})|G\rangle \otimes\left|e_{2}\right\rangle\right) \\
& \left|\Psi_{3^{\prime}}(t)\right\rangle=e^{-\Gamma t}|B\rangle \otimes\left|g_{2}\right\rangle
\end{aligned}
$$

where $|G\rangle=\left|g_{1} g_{3}\right\rangle$ and

$$
|D\rangle=\frac{\left|e_{1} g_{3}\right\rangle+\left|g_{1} e_{3}\right\rangle}{\sqrt{2}} ;|B\rangle=\frac{\left|e_{1} g_{3}\right\rangle-\left|g_{1} e_{3}\right\rangle}{\sqrt{2}}
$$

As is seen from the third equation in 72 the bright state of the two-qubit system decays independently on the presence of the second (central) qubit. However, the decay of the dark state can be revealed only through its entanglement with the second qubit [12. In fact, the interaction between second qubit and the dark state formed by two edge qubits gives rise to the vacuum Rabi oscillations which are shown in (37), (38), and in Fig.4 Fig.5.

\section{A. Relation between qubits amplitudes and collective states}

Here, we show how the qubits amplitudes $\beta_{n}(t)$ are related to collective wave functions $\left|\Psi_{i}(t)\right\rangle(52)$. We write the dynamic wave function $|\Psi(t)\rangle(50)$ as a decomposition over the collective states $(52)$.

$$
|\Psi(t)\rangle=\sum_{i=1}^{3} A_{i}\left|\Psi_{i}(t)\right\rangle=\sum_{i, n=1}^{3} A_{i} e^{-i \bar{E}_{i} t} \alpha_{n}^{(i)}|n\rangle
$$

From (50) we obtain:

$$
\beta_{n}(t)=\sum_{i=1}^{3} e^{-i \bar{E}_{i} t} A_{i} \alpha_{n}^{(i)}
$$

with the initial conditions

$$
\begin{gathered}
\beta_{n_{0}}(0)=\sum_{i=1}^{3} A_{i} \alpha_{n_{0}}^{(i)}=1 \\
\beta_{n}(0)=\sum_{i=1}^{3} A_{i} \alpha_{n}^{(i)}=0 ; n \neq n_{0}
\end{gathered}
$$

where $n_{0}$ is the sequence number of excited qubit.

The probability amplitude $\left|\beta_{n}(t)\right|^{2}$ can directly be expressed in terms of collective state wave functions $\left|\Psi_{i}(t)\right\rangle$.

$$
\left|\beta_{n}(t)\right|^{2}=\left\langle n\left|\left(\sum_{i, j=1}^{3} A_{i} A_{j}^{*}\left|\Psi_{i}\right\rangle\left\langle\Psi_{j}\right|\right)\right| n\right\rangle
$$

From linear algebraic equations 76 we can find coefficients $A_{i}$ and, therefore, restore the qubits' amplitudes $\beta_{n}(t)$. However, if the number of qubits is large, this procedure is not convenient for computer simulations. The main reason is that it requires first, the calculation of $N$ complex energies from determinant of $N \times N$ matrix analogous to 55 , second, the calculations of $\alpha_{n}^{(i)}$ from non linear conditions (57) and (58), and third, the solution of a system of $N$ linear algebraic equations analogous to (76). Every of these three steps is not simple from a mathematical point of view. It is more convenient to directly compute the qubits amplitudes $\beta_{n}(t)$ from a set of the linear differential equations (16), which allow us to completely avoid all three steps we mentioned above.

Nevertheless, we should like to mention some interesting consequences that follow from equations 775 , 766, (77). First, from (75) we see that the dark states $\left(\operatorname{Im} \bar{E}_{i}=0\right)$ contribute to $\beta_{n}(t)$, even though they do not contribute to the spectrum of the photon emission. Second, it follows from $(77)$ that if specific collective state $\left|\Psi_{i}(t)\right\rangle$ does not contain qubit state $|n\rangle$, then this collective state does not take part in the formation of the dynamics of the qubits amplitudes $\beta_{n}(t)$. As was shown above (see (67)) the state $\left|\Psi_{3}(t)\right\rangle$ does not contain the qubit state $|2\rangle$. Therefore, independently on the value of $k d$, only two collective states $\left|\Psi_{1}(t)\right\rangle$ and $\left|\Psi_{2}(t)\right\rangle$ take part in the formation of the dynamics of the qubits amplitudes $\beta_{2}(t)$.

\section{THREE NON-IDENTICAL QUBITS}

Here, we consider a system in which all three qubits are identical except for the frequency of a second qubit which has a different value $\Omega_{0}$. For this case, we rewrite the equations (12), (13), and (14).

$$
\begin{aligned}
& \frac{d \beta_{1}}{d t}=-\frac{\Gamma}{2} \beta_{1}(t)-i \frac{\delta \Omega}{2} \beta_{1}(t) \\
& -\beta_{2}(t) \frac{1}{2}\left(\frac{\Omega_{0}}{\Omega}\right)^{1 / 2} \Gamma e^{i k_{0} d}-\frac{\Gamma}{2} \beta_{3}(t) e^{2 i k d}
\end{aligned}
$$




$$
\begin{gathered}
\frac{d \beta_{2}}{d t}=-\frac{\Gamma}{2} \beta_{2}(t)+i \frac{\delta \Omega}{2} \beta_{2}(t) \\
-\frac{1}{2}\left(\frac{\Omega}{\Omega_{0}}\right)^{1 / 2} \Gamma e^{i k d}\left(\beta_{1}(t)+\beta_{3}(t)\right) \\
\frac{d \beta_{3}}{d t}=-\frac{\Gamma}{2} \beta_{3}(t)-i \frac{\delta \Omega}{2} \beta_{3}(t)-\beta_{1}(t) \frac{\Gamma}{2} e^{2 i k d} \\
-\beta_{2}(t) \frac{1}{2}\left(\frac{\Omega_{0}}{\Omega}\right)^{1 / 2} \Gamma e^{i k_{0} d}
\end{gathered}
$$

where $\delta \Omega=\Omega-\Omega_{0}, k=\frac{\Omega}{v_{g}}, k_{0}=\frac{\Omega_{0}}{v_{g}}$

Characteristic roots can be found from the determinant of equations (78), (79), and 80).

$$
\begin{aligned}
& \lambda_{1}=-\frac{\Gamma}{2}\left(1+\frac{1}{2} e^{2 i k d}\right) \\
& -\frac{\Gamma}{4} e^{i k d} \sqrt{e^{2 i k d}+8 e^{i\left(k_{0}-k\right) d}+4 i \frac{\delta \Omega}{\Gamma}-4\left(\frac{\delta \Omega}{\Gamma}\right)^{2} e^{-2 i k d}}
\end{aligned}
$$

$$
\begin{aligned}
& \lambda_{2}=-\frac{\Gamma}{2}\left(1+\frac{1}{2} e^{2 i k d}\right) \\
& +\frac{\Gamma}{4} e^{i k d} \sqrt{e^{2 i k d}+8 e^{i\left(k_{0}-k\right) d}+4 i \frac{\delta \Omega}{\Gamma}-4\left(\frac{\delta \Omega}{\Gamma}\right)^{2} e^{-2 i k d}}
\end{aligned}
$$

$$
\lambda_{3}=\frac{\Gamma}{2} e^{i 2 k d}-\frac{\Gamma}{2}-i \frac{\delta \Omega}{2}
$$

The calculations show that equations 81 and 82 provide no dark states $\left(\operatorname{Re}\left(\lambda_{1,2}\right)=0\right)$ if $\delta \Omega$ is not equal to zero. The quantities $\operatorname{Re}\left(\lambda_{1,2}\right)$ are always negative at any $k d$. The exception is the third root $\lambda_{3}$, with real part being equal to zero for $k d=n \pi$. Therefore, if the root $\lambda_{3}$ does not contribute to the dynamics of qubits, then at any $k d$, the qubits' amplitudes will decay to zero with the rate being dependent on $\delta \Omega$.

The dependence of real parts of $\lambda_{n}$ on $k d$ is shown in Fig 7 for $\delta \Omega=\Gamma$.

If the second (central) qubit is excited, the amplitudes of the first and the third qubit are the same $\beta_{1}(t)=$ $\beta_{3}(t) \equiv \beta(t)$. Then three equations $(78),(79)$, and 800 reduce to two equations, for $\beta_{2}$ and $\beta$.

$$
\frac{d \beta}{d t}=-\frac{\Gamma}{2}\left(1+e^{2 i k d}+i \frac{\delta \Omega}{\Gamma}\right) \beta(t)-\beta_{2}(t) \frac{\Gamma}{2}\left(\frac{\Omega_{0}}{\Omega}\right)^{1 / 2} e^{i k_{0} d}
$$

$$
\frac{d \beta_{2}}{d t}=-\frac{\Gamma}{2}\left(1-i \frac{\delta \Omega}{\Gamma}\right) \beta_{2}(t)-\beta(t) \Gamma\left(\frac{\Omega}{\Omega_{0}}\right)^{1 / 2} e^{i k d}
$$

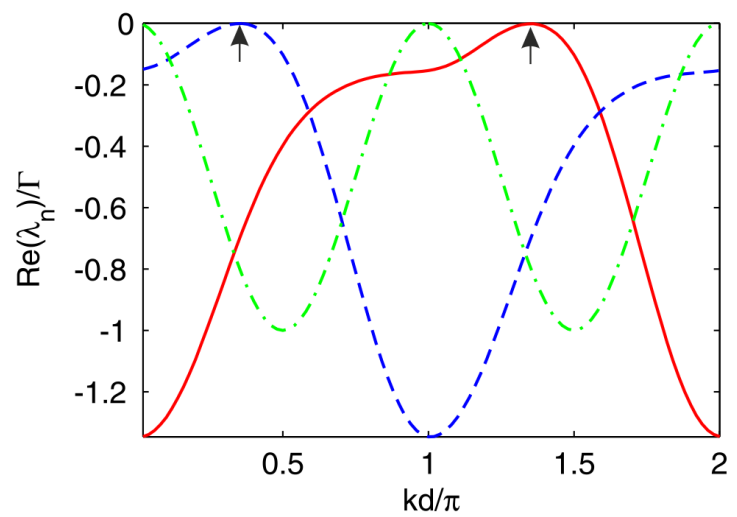

FIG. 7: Dependence of $\operatorname{Re}\left(\lambda_{n}\right) / \Gamma$ on $k d$ for $\delta \Omega=\Gamma$. Solid (red) line relates to $\lambda_{1}$, dashed (blue) line relates to $\lambda_{2}$, and dashed-dotted (green) line relates to $\lambda_{3}$. The arrows show the points of deep subradiant states where $\operatorname{Re}\left(\lambda_{1,2}\right) / \Gamma \approx-0.001$

The characteristic roots of these equations are equal to $\lambda_{1}$ and $\lambda_{2}$ given above in equations (81) and $(82)$.

The solution of equations (84) and (85). with account for initial conditions for the amplitudes, $\beta_{2}(0)=$ $1, \beta(0)=0$ and their time derivatives

$$
\begin{gathered}
\left.\frac{d \beta}{d t}\right|_{t=0}=-\frac{\Gamma}{2}\left(\frac{\Omega_{0}}{\Omega}\right)^{1 / 2} e^{i k_{0} d} \\
\left.\frac{d \beta_{2}}{d t}\right|_{t=0}=-\frac{\Gamma}{2}\left(1-i \frac{\delta \Omega}{\Gamma}\right)
\end{gathered}
$$

is similar to 26

$$
\begin{aligned}
& \beta_{2}(t)=b_{1} e^{\lambda_{1} t}+b_{2} e^{\lambda_{2} t} \\
& \beta(t)=a_{1} e^{\lambda_{1} t}+a_{2} e^{\lambda_{2} t}
\end{aligned}
$$

with

$$
\begin{gathered}
b_{1}=-\frac{\frac{\Gamma}{2}\left(1-i \frac{\delta \Omega}{\Gamma}\right)+\lambda_{2}}{\lambda_{1}-\lambda_{2}} \\
b_{2}=\frac{\frac{\Gamma}{2}\left(1-i \frac{\delta \Omega}{\Gamma}\right)+\lambda_{1}}{\lambda_{1}-\lambda_{2}} \\
a_{1}=-\frac{\Gamma}{2}\left(\frac{\Omega_{0}}{\Omega}\right)^{1 / 2} \frac{e^{i k_{0} d}}{\lambda_{1}-\lambda_{2}} \\
a_{2}=\frac{\Gamma}{2}\left(\frac{\Omega_{0}}{\Omega}\right)^{1 / 2} \frac{e^{i k_{0} d}}{\lambda_{1}-\lambda_{2}}
\end{gathered}
$$

Using the detuning $\delta \Omega$ as the external parameter we can control the decay rates of the qubits amplitudes. This is shown in Fig 8 for several values of the detuning $\delta \Omega$ with the second qubit being initially excited. In the case 
shown in Fig, the decay rates are governed by real parts of two roots, $\lambda_{1}$ and $\lambda_{2}$ with $\left|R e \lambda_{1}\right| \ll\left|R e \lambda_{2}\right|$. As $\delta \Omega / \Gamma$ increases from 0 to $1,\left|\operatorname{Re} \lambda_{1}\right|$ decreases from 1.5 to 1.346 while $\left|R e \lambda_{2}\right|$ increases from 0 to 0.154 . Even though the first root tends to slow down the decay rate as $\delta \Omega / \Gamma$ increases, its influence for $t \gg \Gamma$ becomes negligible, so that the main contribution to the decay rate for large times comes from the second root which speeds up the decay rate as $\delta \Omega / \Gamma$ increases.

Therefore, the greater is $\delta \Omega$, the more is its influence on the qubit decay rates.
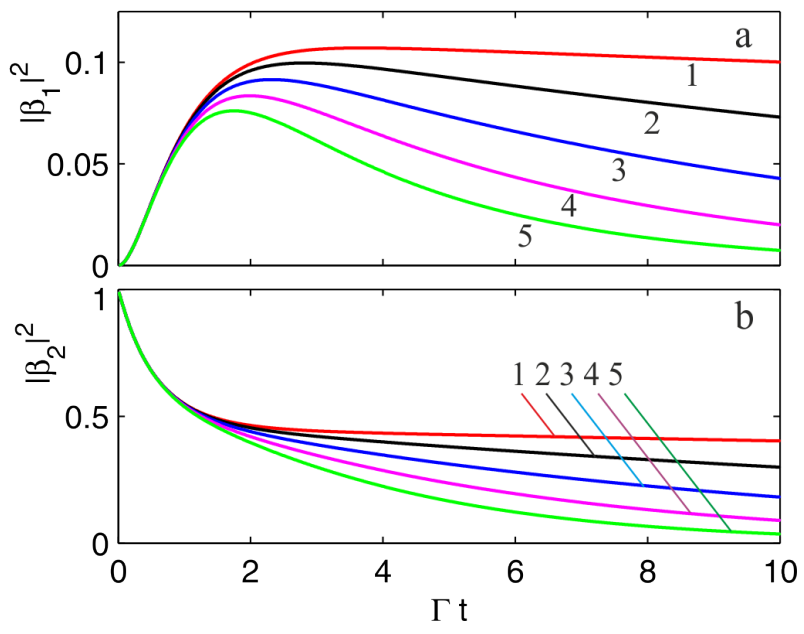

FIG. 8: Dependence of the decay rates on the detuning $\delta \Omega$ for initially excited second qubit. $k d=2 \pi$. (a)-the decay rates of the first(third) qubit; (b) the decay rates of the second qubit. The numbers in the plots correspond to different detunings: $1-\delta \Omega=0.2 \Gamma ; 2-\delta \Omega=0.4 \Gamma ; 3-\delta \Omega=0.6 \Gamma ; 4-\delta \Omega=$ $0.8 \Gamma ; 5-\delta \Omega=\Gamma$.

If the first qubit is initially excited the solution is similar to 33 :

$$
\begin{aligned}
& \beta_{1}(t)=\frac{b_{2}}{2} e^{\lambda_{1} t}+\frac{b_{1}}{2} e^{\lambda_{2} t}+\frac{1}{2} e^{\lambda_{3} t} \\
& \beta_{2}(t)=a_{1}\left(e^{\lambda_{1} t}-e^{\lambda_{2} t}\right) \\
& \beta_{3}(t)=\frac{b_{2}}{2} e^{\lambda_{1} t}+\frac{b_{1}}{2} e^{\lambda_{2} t}-\frac{1}{2} e^{\lambda_{3} t}
\end{aligned}
$$

where $\lambda_{1}, \lambda_{2}, \lambda_{3}$, and $a_{1}, b_{1}, b_{2}$ are given in (81), (82), 83 , and 89,90 .

For this case, we show in Fig. 9 the temporal behavior of qubits amplitudes for several values of the detuning $\delta \Omega$. In the absence of detuning (the panel $a$ ) we obtain the result shown in Fig 3 . However, for non zero detuning the amplitudes of the first and the third qubits decay to 0.25 , while the second qubit decays to zero. This difference can be explained by the influence of dark states (the last terms in the expressions for $\left.\beta_{(} t\right)$ and $\beta_{3}(t)$ in $(91)$ ). In general, as the detuning increases the decay rates increase as well.

The subsystem consisting of a central qubit and the symmetric qubit array can be described as an analogue

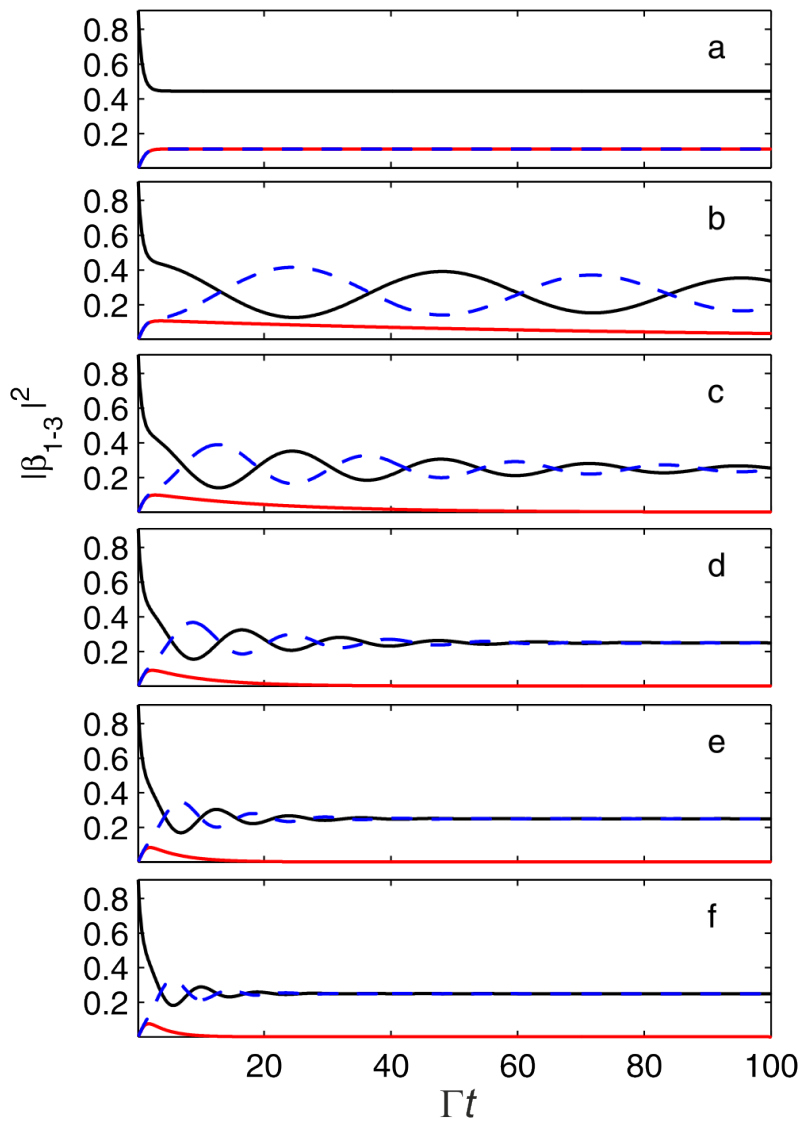

FIG. 9: Dependence of the decay rates on the detuning $\delta \Omega$ for initially excited the first qubit. $k d=2 \pi, \Gamma / \Omega=0.001$. Every panel from $a$ to $f$ shows the decay rates of the the first (black, solid line), second (red, solid line), and third (blue, dashed line) qubit, respectively. From top $(a)$ to bottom $(f)$ the quantity $\delta \Omega / \Gamma$ is varied from $0(a)$ to $1(f)$ with the increment being equal to 0.2

to a cavity QED system. In this description, the central qubit acts as a two-level atom and the symmetric qubit array mimics a high-finesse cavity, with the qubits array acting as an atomic mirror [11. It seems that this analogy is supported by Fig 3 where two dark states 70 of the whole system prevent the qubit amplitudes from the damping. If this analogy worked we would expect that the detuning of a central qubit would not lead to any damping at all. In real cavity the qubit which is large detuned from the cavity resonance cannot exchange its energy with the cavity via real photons. However, as is seen from Fig,8, a full analogy between resonance cavity and the qubit array does not exist. Even a small detuning of the central qubit from the frequency of the qubit array results in the disruption of the dark states that, in turn, leads to the damping of the qubits amplitudes. 


\section{A. Spectral density of spontaneous photon emission}

For the calculation of the spectral density of photon emission we use the equation 10 together with the substitution of $\beta_{n}(t)$ from equations 15 .

$$
\begin{aligned}
& \gamma_{k}(t)=-i g_{k} e^{i k d} \int_{0}^{t} \bar{\beta}_{1}\left(t^{\prime}\right) e^{i\left(\Omega-\Omega_{0}\right) t^{\prime} / 2} e^{i(\omega-\Omega) t^{\prime}} d t^{\prime} \\
& -i g_{k} \int_{0}^{t} \bar{\beta}_{2}\left(t^{\prime}\right) e^{-i\left(\Omega-\Omega_{0}\right) t^{\prime} / 2} e^{i\left(\omega-\Omega_{0}\right) t^{\prime}} d t^{\prime} \\
& -i g_{k} e^{-i k d} \int_{0}^{t} \bar{\beta}_{3}\left(t^{\prime}\right) e^{i\left(\Omega-\Omega_{0}\right) t^{\prime} / 2} e^{i(\omega-\Omega) t^{\prime}} d t^{\prime}
\end{aligned}
$$

where $k d=\frac{\omega}{v_{g}} d, g_{k}=\left(\frac{v_{g} \Gamma}{2 L}\right)^{1 / 2}$.

The amplitudes $\bar{\beta}_{n}(t)$ in $(92)$ are just the amplitudes $\beta_{n}(t)$ given above in 88 and $(91)$.

Below we show two plots which demonstrate how the spectral density of photon emission depends on the detuning $\delta \Omega$. The dependencies of spectral density on detuning are shown in Fig, 10 for $k d=2 \pi$ and in Fig 11 for $k d=3 \pi / 2$, respectively. In both cases the second qubit was initially excited.

If $\delta \Omega=0$ and $k d=2 \pi$ we see a single Lorentzian peak (Fig, 10,a)) with the width equal to $3 \Gamma$ (see also 45). If $\delta \Omega \neq 0$ a Lorentzian peak splits into two narrow peaks. The distance between the peaks, $\Delta \omega$ is approximately equal to $\left(\left|\operatorname{Im} \lambda_{1}\right|+\left|\operatorname{Im} \lambda_{2}\right|\right)$. The width of the peaks is determined by the root with a lowest real part. In the case shown in Fig 10(b-f) the width of the peaks and their height are approximately equal to $\left|\operatorname{Re} \lambda_{2}\right|$ and $\Omega /\left|R e \lambda_{2}\right|$, respectively. To estimate the order of these quantities we have found numerically the range of variation for $R e \lambda_{2}$, $I m \lambda_{1}$, and $I m \lambda_{2}$. As $\delta \Omega / \Gamma$ increases from 0.2 to 1 , the quantities $\operatorname{Re} \lambda_{2}, \operatorname{Im} \lambda_{1}$, and $\operatorname{Im} \lambda_{2}$ vary from $-0.006 \Gamma$ to $-0.154 \Gamma$, from $+0.028 \Gamma$ to $+0.202 \Gamma$, and from $-0.032 \Gamma$ to $-0.205 \Gamma$, respectively.

If $\delta \Omega=0$ and $k d=3 \pi / 2$ there are two similar peaks shown in Fig 11(a) (see also Fig 6(a)). The distance between the peaks and their widths are determined by the roots $\lambda_{1}$ and $\lambda_{2}: \operatorname{Im} \lambda_{1}=-\operatorname{Im} \lambda_{2}=(\sqrt{7} / 4) \Gamma$, $\operatorname{Re} \lambda_{1}=\operatorname{Re} \lambda_{2}=-0.25 \Gamma$ (see Eq. (21)). As $\delta \Omega / \Gamma$ increases from 0.2 to 1 , the quantities $R e \lambda_{1}$ and $R e \lambda_{2}$ are of the same order of magnitude: $R e \lambda_{1}$ varies from $-0.287 \Gamma$ to $-0.399 \Gamma$, and $R e \lambda_{2}$ varies from $-0.213 \Gamma$ to $-0.100 \Gamma$. This behavior is confirmed in Fig 11 (b-f) where the width of the left peak increases, while the width of the right peak decreases. The distance between the peaks are determined by the quantities $\operatorname{Im} \lambda_{1}$ and $\operatorname{Im} \lambda_{2}$ which vary from $-0.708 \Gamma$ to $-0.890 \Gamma$, and from $+0.638 \Gamma$ to $+0.821 \Gamma$, respectively.

We also calculated the temporal behavior of the full probability of the photon emission , $P_{p h}(t)$ as a function

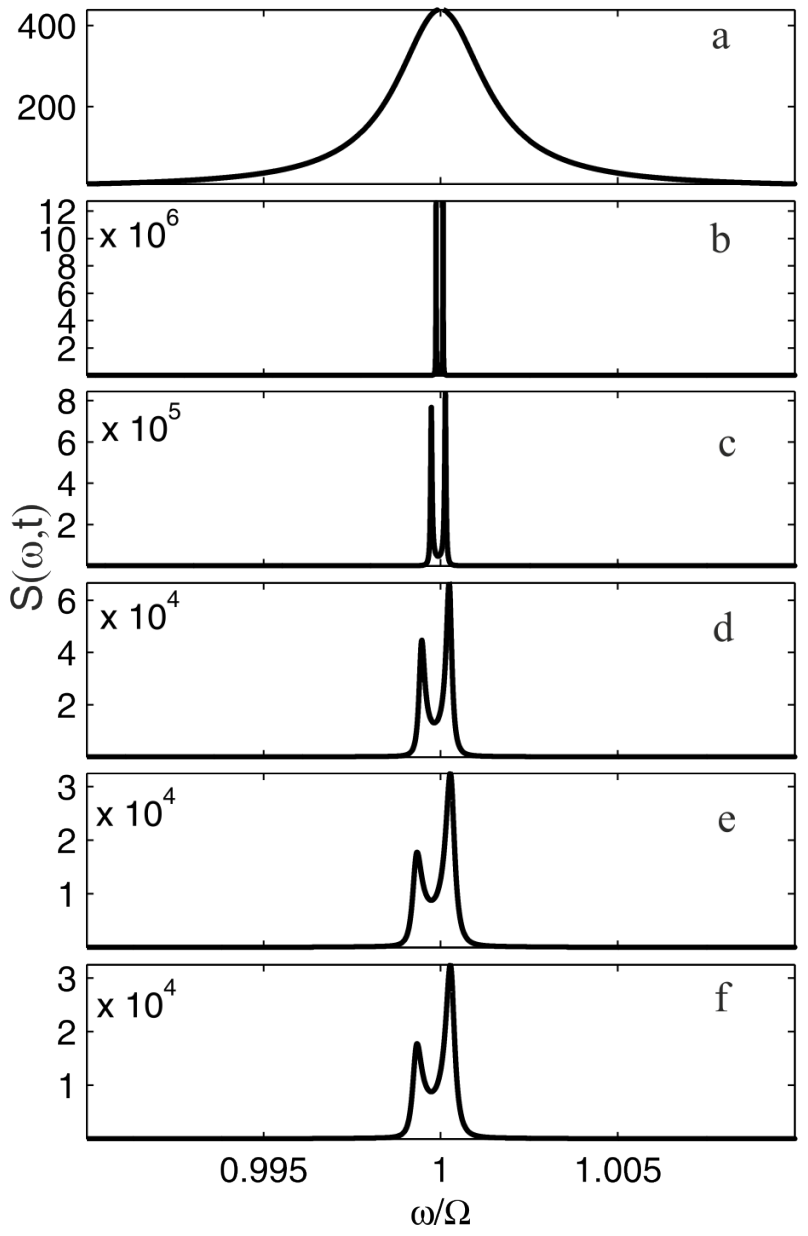

FIG. 10: Dependence of spectral density on the detuning. Second qubit is initially excited. $k d=2 \pi, \Gamma / \Omega=0.001, t \gg$ $1 / \Gamma$. From top (a) to bottom (f) the quantity $\delta \Omega / \Gamma$ is varied from 0 (a) to 1 (f) with the increment being equal to 0.2 .

of the detuning. This dependence is shown in Fig.12. It is evident from the definition (8) that $P_{p h}(0)=0$ and as the time increases it approaches either 1 if all qubits amplitudes damp out to zero, or a constant value if not all qubits decay to zero. Because the detuning influences the rate of the qubits damping, the output rate of the photon emission, $d P_{p h} / d t$ also depends on the detuning. The greater is the detuning, the greater is the rate of the photon emission.

\section{CONCLUSION}

In this paper we have thoroughly investigated the dynamics behavior of qubits amplitudes in 1D chain consisting of three qubits embedded in an open waveguide. Within a single-excitation subspace we have found the evolution of qubits amplitudes if one of the qubits was initially excited. We have shown that even though the dark states do not contribute to the output photon emission, they influence the evolution of qubits amplitudes in 


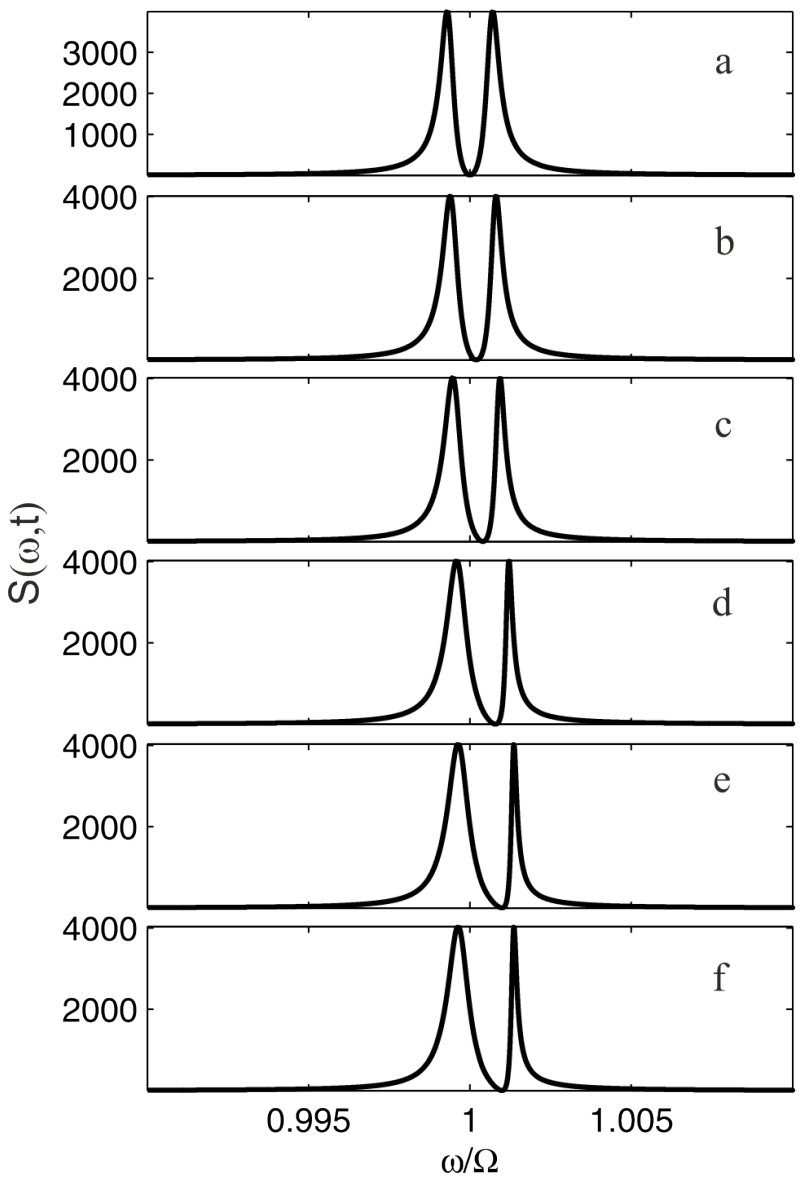

FIG. 11: Dependence of spectral density on the detuning. Second qubit is initially excited. $k d=3 \pi / 2, \Gamma / \Omega=0.001$, $t \gg 1 / \Gamma$. From top (a) to bottom (f) the quantity $\delta \Omega / \Gamma$ is varied from 0 (a) to 1 (f) with the increment being equal to 0.2

that they prevent the qubits amplitudes from decaying to zero. We have found the collective eigenstates of a threequbit system and have shown how the qubits amplitudes can be expressed in terms of the amplitudes of the collective states. We also calculated the spectral density of the output photon emission from three-qubit system and studied its dependence on the $k d$ value. We studied the case when the frequency of the second qubit was different from that of the edge qubits. In this case, the rates of qubits decay crucially depend on the frequency detuning between central and edge qubits. The greater is the detuning, the greater is the rate of the qubits damping.

We hope that this research will prove useful for the development of the efficient control and readout protocols for a few-qubit quantum processor.

\section{Acknowledgments}

Ya. S. G. thanks A. Sultanov for fruitful discussions. The work is supported by the Ministry of Education and

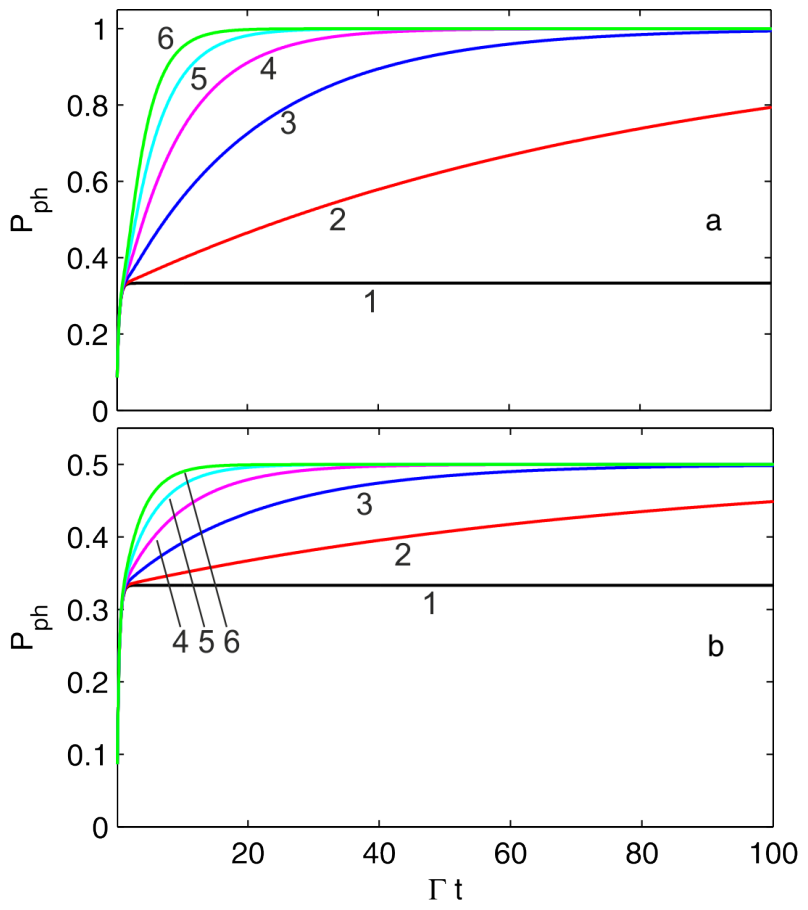

FIG. 12: The probability amplitude of the photon emission. $k d=2 \pi, \Gamma / \Omega=0.001$. (a) the second qubit is initially excited; (b) the first qubit is initially excited. The numbers in the plots correspond to different detunings: $1-\delta \Omega=0$, $2-\delta \Omega=0.2 \Gamma ; 3-\delta \Omega=0.4 \Gamma ; 4-\delta \Omega=0.6 \Gamma ; 5-\delta \Omega=$ $0.8 \Gamma ; 6-\delta \Omega=\Gamma$.

Science of Russian Federation under the project FSUN2020-0004.

\section{Appendix A: Equations for qubits amplitudes in the Wigner-Weisskopf approximation}

We assume that the first and the third qubits are identical $\left(\Omega_{1}=\Omega_{3} \equiv \Omega, g_{k}^{(1)}=g_{k}^{(3)} \equiv g_{k}\right)$ The frequency and the coupling of the second qubit are different $\left(\Omega_{2} \equiv\right.$ $\left.\Omega_{0}, g_{k}^{(2)} \equiv g_{k}^{(0)}\right)$. A distance between central qubit and the edge qubits is equal to $d$. We take the origin in the location of the second qubit: $x_{1}=-d, x_{2}=0, x_{3}=+d$. For this case, we expand the equation $(9)$ as a set of three equations. 


$$
\begin{aligned}
& \frac{d \beta_{1}}{d t}=-\sum_{k} g_{k}^{2} \int_{0}^{t} \beta_{1}\left(t^{\prime}\right) e^{-i\left(\omega_{k}-\Omega\right)\left(t-t^{\prime}\right)} d t^{\prime} \\
& -\sum_{k} g_{k} g_{k}^{(0)} e^{-i k d} e^{i\left(\Omega-\Omega_{0}\right) t} \int_{0}^{t} \beta_{2}\left(t^{\prime}\right) e^{-i\left(\omega_{k}-\Omega_{0}\right)\left(t-t^{\prime}\right)} d t^{\prime} \\
& -\sum_{k} g_{k}^{2} e^{-i 2 k d} \int_{0}^{t} \beta_{3}\left(t^{\prime}\right) e^{-i\left(\omega_{k}-\Omega\right)\left(t-t^{\prime}\right)} d t^{\prime} \\
& \frac{d \beta_{2}}{d t}=-\sum_{k}\left(g_{k}^{(0)}\right)^{2} \int_{0}^{t} \beta_{2}\left(t^{\prime}\right) e^{-i\left(\omega_{k}-\Omega_{0}\right)\left(t-t^{\prime}\right)} d t^{\prime} \\
& -\sum_{k} g_{k}^{(0)} g_{k} e^{i k d} e^{i\left(\Omega_{0}-\Omega\right) t} \int_{0}^{t} \beta_{1}\left(t^{\prime}\right) e^{-i\left(\omega_{k}-\Omega\right)\left(t-t^{\prime}\right)} d t^{\prime} \\
& -\sum_{k} g_{k}^{(0)} g_{k} e^{-i k d} e^{i\left(\Omega_{0}-\Omega\right) t} \int_{0}^{t} \beta_{3}\left(t^{\prime}\right) e^{-i\left(\omega_{k}-\Omega\right)\left(t-t^{\prime}\right)} d t^{\prime} \\
& \frac{d \beta_{3}}{d t}=-\sum_{k} g_{k}^{2} \int_{0}^{t} \beta_{3}\left(t^{\prime}\right) e^{-i\left(\omega_{k}-\Omega\right)\left(t-t^{\prime}\right)} d t^{\prime} \\
& -\sum_{k} g_{k}^{2} e^{i 2 k d} \int_{0}^{t} \beta_{1}\left(t^{\prime}\right) e^{-i\left(\omega_{k}-\Omega\right)\left(t-t^{\prime}\right)} d t^{\prime} \\
& -\sum_{k} g_{k} g_{k}^{(0)} e^{i k d} e^{i\left(\Omega-\Omega_{0}\right) t} \int_{0}^{t} \beta_{2}\left(t^{\prime}\right) e^{-i\left(\omega_{k}-\Omega_{0}\right)\left(t-t^{\prime}\right)} d t^{\prime}
\end{aligned}
$$

According to Wigner-Weisskopf approach we replace $\beta_{1}\left(t^{\prime}\right), \beta_{2}\left(t^{\prime}\right), \beta_{3}\left(t^{\prime}\right)$ in the integrands with $\beta_{1}(t), \beta_{2}(t), \beta_{3}(t)$ and take them out of the integrals.

$$
\begin{aligned}
& \frac{d \beta_{1}}{d t}=-\beta_{1}(t) \sum_{k} g_{k}^{2} I_{k}(\Omega, t) \\
& -\beta_{2}(t) \sum_{k} g_{k} g_{k}^{(0)} e^{-i k d} e^{i\left(\Omega-\Omega_{0}\right) t} I_{k}\left(\Omega_{0}, t\right) \\
& -\beta_{3}(t) \sum_{k} g_{k}^{2} e^{-i 2 k d} I_{k}(\Omega, t) \\
& \frac{d \beta_{2}}{d t}=-\beta_{2}(t) \sum_{k}\left|g_{k}^{(0)}\right|^{2} I_{k}\left(\Omega_{0}, t\right) \\
& -\beta_{1}(t) \sum_{k} g_{k}^{(0)} g_{k} e^{i k d} e^{-i\left(\Omega-\Omega_{0}\right) t} I_{k}(\Omega, t) \\
& -\beta_{3}(t) \sum_{k} g_{k}^{(0)} g_{k} e^{-i k d} e^{-i\left(\Omega-\Omega_{0}\right) t} I_{k}(\Omega, t)
\end{aligned}
$$

$$
\begin{aligned}
& \frac{d \beta_{3}}{d t}=-\beta_{3}(t) \sum_{k} g_{k}^{2} I_{k}(\Omega, t) \\
& -\beta_{1}(t) \sum_{k} g_{k}^{2} e^{i 2 k d} I_{k}(\Omega, t) \\
& -\beta_{2}(t) \sum_{k} g_{k} g_{k}^{(0)} e^{i k d} e^{i\left(\Omega-\Omega_{0}\right) t} I_{k}\left(\Omega_{0}, t\right)
\end{aligned}
$$

where

$$
\begin{aligned}
& I_{k}(\Omega, t)=\int_{0}^{t} e^{-i\left(\omega_{k}-\Omega\right)\left(t-t^{\prime}\right)} d t^{\prime}=\int_{0}^{t} e^{-i\left(\omega_{k}-\Omega\right) \tau} d \tau \\
& \approx \int_{0}^{\infty} e^{-i\left(\omega_{k}-\Omega\right) \tau} d \tau=\pi \delta\left(\omega_{k}-\Omega\right)-i P \cdot v \cdot\left(\frac{1}{\omega_{k}-\Omega}\right)
\end{aligned}
$$

where P.v. is a Cauchy principal value integral.

We can remove oscillating exponents in A4 - A6 with the aid of the substitution

$$
\begin{aligned}
& \beta_{1}(t)=e^{i\left(\Omega-\Omega_{0}\right) t / 2} \bar{\beta}_{1}(t) \\
& \beta_{2}(t)=e^{-i\left(\Omega-\Omega_{0}\right) t / 2} \bar{\beta}_{2}(t) \\
& \beta_{3}(t)=e^{i\left(\Omega-\Omega_{0}\right) t / 2} \bar{\beta}_{3}(t)
\end{aligned}
$$

In addition, we assume the coupling constants $g_{k}$ are even functions of $k\left(g_{k}=g_{-k}\right)$. Then from A4 - A6 we obtain

$$
\begin{aligned}
& \frac{d \bar{\beta}_{1}}{d t}=-\bar{\beta}_{1}(t) \sum_{k} g_{k}^{2} I_{k}(\Omega, t)-i \frac{\Omega-\Omega_{0}}{2} \bar{\beta}_{1}(t) \\
& -\bar{\beta}_{2}(t) 2 \sum_{k>0} g_{k} g_{k}^{(0)} \cos (k d) I_{k}\left(\Omega_{0}, t\right) \\
& -\bar{\beta}_{3}(t) 2 \sum_{k>0} g_{k}^{2} \cos (2 k d) I_{k}(\Omega, t)
\end{aligned}
$$

$$
\begin{aligned}
& \frac{d \bar{\beta}_{2}}{d t}=-\bar{\beta}_{2}(t) \sum_{k}\left(g_{k}^{(0)}\right)^{2} I_{k}\left(\Omega_{0}, t\right)+i \frac{\Omega-\Omega_{0}}{2} \bar{\beta}_{2}(t) \\
& -\bar{\beta}_{1}(t) 2 \sum_{k>0} g_{k}^{(0)} g_{k} \cos (k d) I_{k}(\Omega, t) \\
& -\bar{\beta}_{3}(t) 2 \sum_{k>0} g_{k}^{(0)} g_{k} \cos (k d) I_{k}(\Omega, t)
\end{aligned}
$$

$$
\begin{aligned}
& \frac{d \bar{\beta}_{3}}{d t}=-\bar{\beta}_{3}(t) \sum_{k} g_{k}^{2} I_{k}(\Omega, t)-i \frac{\Omega-\Omega_{0}}{2} \bar{\beta}_{3}(t) \\
& -\bar{\beta}_{1}(t) 2 \sum_{k>0} g_{k}^{2} \cos (2 k d) I_{k}(\Omega, t) \\
& -\bar{\beta}_{2}(t) 2 \sum_{k>0} g_{k} g_{k}^{(0)} \cos (k d) I_{k}\left(\Omega_{0}, t\right)
\end{aligned}
$$


The next step is to relate the coupling constants $g_{k}$ to the qubit decay rate of spontaneous emission into waveguide mode. In accordance with Fermi golden rule we define the qubit decay rates by the following expressions:

$$
\begin{gathered}
\Gamma=2 \pi \sum_{k} g_{k}^{2} \delta\left(\omega_{k}-\Omega\right) \\
\Gamma_{0}=2 \pi \sum_{k}\left(g_{k}^{(0)}\right)^{2} \delta\left(\omega_{k}-\Omega_{0}\right)
\end{gathered}
$$

where

$$
g_{k}=\sqrt{\frac{\omega_{k} D^{2}}{2 \hbar \varepsilon_{0} V}}
$$

$D$ is the matrix element of the qubit's dipole moment operator, $V$ is the effective volume where the interaction between qubit and electromagnetic field takes place. For $1 \mathrm{D}$ case, a summation over $k$ is replaced by the integration over $\omega$ in accordance with the prescription:

$$
\sum_{k} \Rightarrow \frac{L}{2 \pi} \int_{-\infty}^{\infty} d k=\frac{L}{2 \pi} 2 \int_{0}^{\infty} d|k|=\frac{L}{\pi v_{g}} \int_{0}^{\infty} d \omega_{k}
$$

where $L$ is a length of the waveguide, and we assumed a linear dispersion law, $\omega_{k}=v_{g} k$. The application of A15 to, for example, A12, allows the relation between a coupling constant $g_{k}$ and the decay rate $\Gamma$.

$$
g_{\Omega}=\sqrt{\frac{\Omega D^{2}}{2 \hbar \varepsilon_{0} V}}=\left(\frac{v_{g} \Gamma}{2 L}\right)^{1 / 2}
$$

Therefore, we may relate the coupling constants $g_{\Omega}^{(0)}, g_{\Omega_{2}}^{(0)}, g_{\Omega_{0}}$ with their respective decay rates.

$$
\begin{gathered}
g_{\Omega}^{(0)}=\sqrt{\frac{\Omega D_{0}^{2}}{2 \hbar \varepsilon_{0} V}}=\left(\frac{\Omega}{\Omega_{0}}\right)^{1 / 2}\left(\frac{v_{g}}{2 L} \Gamma_{0}\right)^{1 / 2} \\
g_{\Omega_{0}}=\sqrt{\frac{\Omega_{0} D^{2}}{2 \hbar \varepsilon_{0} V}}=\left(\frac{\Omega_{0}}{\Omega}\right)^{1 / 2}\left(\frac{v_{g}}{2 L} \Gamma\right)^{1 / 2} \\
g_{\Omega_{0}}^{(0)}=\sqrt{\frac{\Omega_{0} D_{0}^{2}}{2 \hbar \varepsilon_{0} V}}=\left(\frac{v_{g}}{2 L} \Gamma_{0}\right)^{1 / 2}
\end{gathered}
$$

Now we can calculate the different terms in A9 A11. We begin with the sum in the first line in A9.

$$
\begin{aligned}
& \sum_{k} g_{k}^{2} I_{k}(\Omega, t)=\sum_{k} g_{k}^{2}\left(\pi \delta\left(\omega_{k}-\Omega\right)-i P . v \cdot\left(\frac{1}{\omega_{k}-\Omega}\right)\right) \\
& =\frac{\Gamma}{2}-i P . v \cdot \sum_{k}\left(\frac{g_{k}^{2}}{\omega_{k}-\Omega}\right) \approx \frac{\Gamma}{2}
\end{aligned}
$$

where $\Gamma$ is given in $\mathrm{A} 12$.

The second term in (A20) gives rise to the shift of the qubit frequency. Therefore, we incorporate it in the renormalized qubit frequency and will not write it explicitly any more. The sum in the second line in A9 is calculated as follows:

$$
\begin{aligned}
& 2 \sum_{k>0} g_{k} g_{k}^{(0)} \cos (k d) I_{k}\left(\Omega_{0}, t\right)= \\
& =\frac{L}{v_{g}} \int_{0}^{\infty} g_{k}^{(0)} g_{k} \cos (k d) \delta\left(\omega_{k}-\Omega_{2}\right) d \omega_{k} \\
& -2 i P . v . \sum_{k>0}\left(\frac{g_{k} g_{k}^{(0)} \cos (k d)}{\omega_{k}-\Omega_{0}}\right) \\
& =\frac{L}{v_{g}} g_{\Omega_{0}}^{(0)} g_{\Omega_{0}} \cos \left(k_{0} d\right)-i \frac{L}{v_{g} \pi} g_{\Omega_{0}} g_{\Omega_{0}}^{(0)} P \cdot v \cdot \int_{0}^{\infty} d \omega \frac{\cos \left(\frac{\omega}{v_{g}} d\right)}{\omega-\Omega_{0}}
\end{aligned}
$$

For principle value integral in A21 we obtain with a good accuracy (see Appendix B):

$$
\text { P.v. } \int_{0}^{\infty} d \omega \frac{\cos \left(\frac{\omega}{v_{g}} d\right)}{\omega-\Omega} \approx-\pi \sin \left(\frac{\Omega}{v_{g}} d\right)=-\pi \sin \left(k_{\Omega} d\right)
$$

Therefore, we finally obtain:

$$
\begin{aligned}
& 2 \sum_{k>0} g_{k} g_{k}^{(0)} \cos (k d) I_{k}\left(\Omega_{0}, t\right)=\frac{L}{v_{g}} g_{\Omega_{0}}^{(0)} g_{\Omega_{0}} e^{i k_{0} d} \\
& =\frac{1}{2}\left(\frac{\Omega_{0}}{\Omega}\right)^{1 / 2} \sqrt{\Gamma \Gamma_{0}} e^{i k_{0} d}
\end{aligned}
$$

Similar calculations give for the last sum in A9:

$$
2 \sum_{k>0} g_{k}^{2} \cos (2 k d) I_{k}(\Omega, t)=\frac{\Gamma}{2} e^{2 i k d}
$$

Collecting together A20, A23, and A24 we write the final form of equation (A9):

$$
\begin{aligned}
& \frac{d \bar{\beta}_{2}}{d t}=-\frac{\Gamma_{0}}{2} \bar{\beta}_{2}(t)+i \frac{\Omega-\Omega_{0}}{2} \bar{\beta}_{2}(t) \\
& -\frac{1}{2}\left(\frac{\Omega}{\Omega_{0}}\right)^{1 / 2} \sqrt{\Gamma \Gamma_{0}} e^{i k d}\left(\bar{\beta}_{1}(t)+\bar{\beta}_{3}(t)\right)
\end{aligned}
$$


Similar calculations for the equations A10 and A11 yield the following result:

$$
\begin{gathered}
\frac{d \bar{\beta}_{2}}{d t}=-\frac{\Gamma_{0}}{2} \bar{\beta}_{2}(t)+i \frac{\Omega-\Omega_{0}}{2} \bar{\beta}_{2}(t) \\
-\frac{1}{2}\left(\frac{\Omega}{\Omega_{0}}\right)^{1 / 2} \sqrt{\Gamma \Gamma_{0}} e^{i k d}\left(\bar{\beta}_{1}(t)+\bar{\beta}_{3}(t)\right) \\
\frac{d \bar{\beta}_{3}}{d t}=-\frac{\Gamma}{2} \bar{\beta}_{3}(t)-i \frac{\Omega-\Omega_{0}}{2} \bar{\beta}_{3}(t)-\bar{\beta}_{1}(t) \frac{\Gamma}{2} e^{2 i k d} \\
-\bar{\beta}_{2}(t) \frac{1}{2}\left(\frac{\Omega_{0}}{\Omega}\right)^{1 / 2} \sqrt{\Gamma \Gamma_{0}} e^{i k_{0} d}
\end{gathered}
$$

\section{Appendix B: Proof of equation A22}

The integral

$$
\int_{0}^{\infty} d \omega \frac{\cos \left(\frac{\omega}{v_{g}} d\right)}{\omega-\Omega}=\int_{0}^{\infty} d x \frac{\cos (a x)}{x-1}
$$

, where $a=k_{\Omega} d$, can be expressed in terms of sine and cosine integrals, $c i$ and si [23]:

$$
\begin{aligned}
& \int_{0}^{\infty} d x \frac{\cos (a x)}{x-1}=-\cos (a) c i(a)-\sin (a)[\operatorname{si}(a)+\pi] \\
& =-\cos (a) C i(a)-\sin (a)\left[\operatorname{Si}(a)+\frac{\pi}{2}\right]
\end{aligned}
$$

where

$$
C i(a)=-\int_{a}^{\infty} \frac{\cos t}{t} d t ; S i(a)=\int_{0}^{a} \frac{\sin t}{t} d t
$$

The integrand in left hand side in (B1) has a singular point at $x=1$ which manifests itself as a singularity of $C i(a)$ at $a \rightarrow 0$ in right hand side in (B1). Therefore, we calculate integral (B1) as Cauchy principal value integral.

$$
\text { P.v. } \int_{-\infty}^{\infty} \frac{\cos a t}{t} d t=\lim _{R \rightarrow \infty} \lim _{\varepsilon \rightarrow 0}\left(\int_{-R}^{-\varepsilon} \frac{\cos a t}{t} d t+\int_{\varepsilon}^{R} \frac{\cos a t}{t} d t\right)
$$

P.v. $\int_{-\infty}^{\infty} \frac{\sin a t}{t} d t=\lim _{R \rightarrow \infty} \lim _{\varepsilon \rightarrow 0}\left(\int_{-R}^{-\varepsilon} \frac{\sin a t}{t} d t+\int_{\varepsilon}^{R} \frac{\sin a t}{t} d t\right)$

Since

$$
\int_{-R}^{-\varepsilon} \frac{\cos a t}{t} d t=-\int_{\varepsilon}^{R} \frac{\cos a t}{t} d t
$$

and

$$
\int_{-R}^{-\varepsilon} \frac{\sin a t}{t} d t=\int_{\varepsilon}^{R} \frac{\sin a t}{t} d t
$$

we obtain

$$
\text { P.v. } \int_{-\infty}^{\infty} \frac{\cos a t}{t} d t=0
$$

$$
\begin{aligned}
& \text { P.v. } \int_{-\infty}^{\infty} \frac{\sin a t}{t} d t=\lim _{R \rightarrow \infty} \lim _{\varepsilon \rightarrow 0}\left(2 \int_{\varepsilon}^{R} \frac{\sin a t}{t} d t\right)= \\
& \lim _{R \rightarrow \infty}\left(2 \int_{0}^{R} \frac{\sin a t}{t} d t\right)=\lim _{R \rightarrow \infty}(2 \operatorname{Si}(a R))=\pi
\end{aligned}
$$

The final result in (B7) is due to the known relation 23]: $\lim _{x \rightarrow \infty} \operatorname{Si}(x)=\frac{\pi}{2}$.

Therefore, we finally obtain

$$
\begin{aligned}
& \text { P.v. } \int_{0}^{\infty} \frac{\cos a x}{x-1} d x \approx \text { P.v. } \int_{-\infty}^{\infty} \frac{\cos a x}{x-1} d x=P . v . \int_{-\infty}^{\infty} \frac{\cos a(t+1)}{t} d t \\
& =\cos a \text { P.v. } \int_{-\infty}^{\infty} \frac{\cos a t}{t} d t-\sin a \text { P.v. } \int_{-\infty}^{\infty} \frac{\sin a t}{t} d t
\end{aligned}
$$

$$
\text { P.v. } \int_{0}^{\infty} \frac{\cos a x}{x-1} d x \approx-\pi \sin a
$$

Below, in Fig 13 we compare the $k d$ - dependence of (B1) with that of (B8). We see a noticeable discrepancy for $k d<\pi / 4$. For $k d>\pi / 2$ two curves are almost identical. 


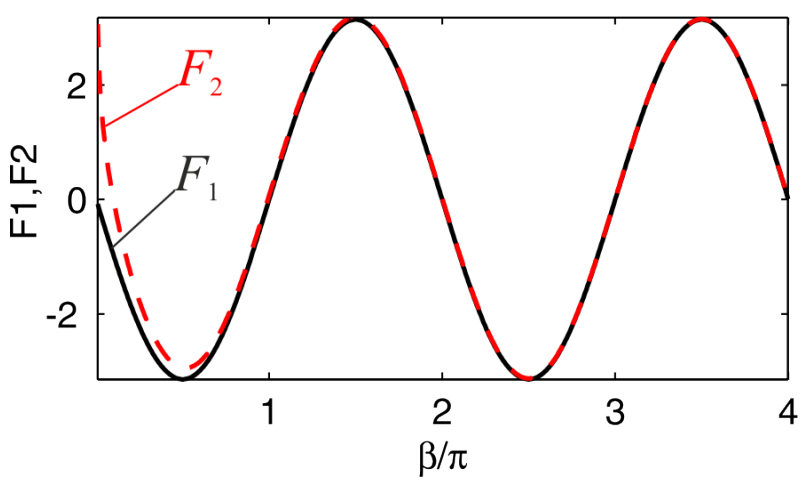

FIG. 13: Comparison of principle value $F_{1}$ (right hand side of (B8) with the "exact" expression $F_{2}$ (right hand side of (B1)).

[1] O. Astafiev, A. M. Zagoskin, A. A. Abdumalikov, Yu. A. Pashkin, T. Yamamoto, K. Inomata, Y. Nakamura, and J. S. Tsai, Resonance Fluorescence of a Single Artificial Atom. Science 327, 840 (2010).

[2] I.-C. Hoi, C. M. Wilson, G. Johansson, T. Palomaki, B. Peropadre, and P. Delsing, Phys. Demonstration of a Single-Photon Router in the Microwave Regime. Rev. Lett. 107, 073601 (2011).

[3] A. F. van Loo, A. Fedorov, K. Lalumi'ere, B. C. Sanders, A. Blais, and A. Wallra, Photon-Mediated Interactions Between Distant Artificial Atoms. Science 342, 1494 (2013).

[4] D. Roy, C. M. Wilson, and O. Firstenberg, Strongly interacting photons in one-dimensional continuum Rev. Mod. Phys. 89, 021001 (2017).

[5] X. Gu, A. F. Kockum, A. Miranowicz, Yu- xi Liu, and F. Nori, Microwave photonics with superconducting quantum circuits. Phys. Rep. 718-719, 1 (2017).

[6] S. N. Shevchenko, Mesoscopic Physics Meets Quantum Engineering. World Scientific, Singapore (2019).

[7] A. Albrecht, L. Henriet, A. Asenjo-Garcia, P. B. Dieterle, O. Painter, and D. E. Chang, Subradiant states of quantum bits coupled to a one-dimensional waveguide, New J. Phys. 21, 025003 (2019).

[8] Y.-X. Zhang and K. Molmer, Theory of Subradiant States of a One-Dimensional Two-Level Atom Chain, Phys. Rev. Lett. 122, 203605 (2019).

[9] J. Ruostekoski and J. Javanainen, Arrays of strongly coupled atoms in a one-dimensional waveguide. Phys. Rev. A 96, 033857 (2017).

[10] K. Lalumi'ere, B. C. Sanders, A. F. van Loo, A. Fedorov, A. Wallraff, and A. Blais, Input-output theory for waveguide QED with an ensemble of inhomogeneous atoms. Phys. Rev. A 88, 043806 (2013).

[11] D. E. Chang, L. Jiang, A. V. Gorshkov, and H. J. Kimble, Cavity QED with atomic mirrors. New J. Phys. 14, 063003 (2012).

[12] M. Mirhosseini, E. Kim, X. Zhang, A. Sipahigil, P. B. Dieterle, A. J. Keller,A. Asenjo-Garcia, D. E. Chang, and O. Painter, Cavity quantum electrodynamics with atom-like mirrors. Nature 569, 692 (2019).

[13] J. D. Brehm, A. N. Poddubny, A. Stehli, T. Wolz, H.
Rotzinger, and A. V. Ustinov, Waveguide bandgap engineering with an array of superconducting qubits, npj Quantum Materials 6, 10 (2021).

[14] A. F. van Loo, A. Fedorov, K. Lalumi'ere, B. C. Sanders, A. Blais, and A. Wallraff Photon-mediated interactions between distant artificial atoms. Science 342, 1494 (2014).

[15] E. Barnes, C. Arenz, A. Pitchford, and S. E. Economou, Fast microwave-driven three-qubit gates for cavitycoupled superconducting qubits. Phys. Rev B 96, 024504 (2017).

[16] L. T. Kenfack, M. Tchoffo, G. C. Fouokeng, and L. C. Fai, Dynamical evolution of entanglement of a three-qubit system driven by a classical environmental colored noise Quant. Inf. Process 17, 76 (2018).

[17] T. S. Tsoi and C. K. Law, Quantum interference effects of a single photon interacting with an atomic chain inside a one-dimensional waveguide. Phys. Rev. A 78, 063832 (2008).

[18] Yu Zhou, Zh. Zhang, Z. Yin, S. Huai, X. Gu, X. Xu, J. Allcock, F. Liu, G.Xi, Q. Yu, H. Zhang, M. Zhang, H. Li, X. Song, Zh. Wang, D. Zheng, Sh. An, Y. Zheng, and Sh. Zhang, Rapid and Unconditional Parametric Reset Protocol for Tunable Superconducting Qubits. ArXive: 2103.11315 [quant-ph] (2021).

[19] P. Magnard,, Ph. Kurpiers, B. Royer,, T, Walter, J.C. Besse,, S. Gasparinetti,, M. Pechal,, J. Heinsoo,, S. Storz, A. Blais, and, A, Wallraff, Fast and Unconditional All-Microwave Reset of a Superconducting Qubit. Phys. Rev. Lett. 121, 060502 (2018).

[20] R. H. Lehmberg, Radiation from an N-Atom System. I. General Formalism. Phys. Rev. A 2, 883 (1970).

[21] F. Keck, H. J. Korsch, and S. Mossmann, Unfolding a diabolic point: a generalized crossing scenario. J. Phys. A: Math. Gen. 36, 2125 (2003).

[22] D. C. Brody, Biorthogonal quantum mechanics. J. Phys. A: Math. Theor. 47, 035305 (2014).

[23] A. P. Prudnikov, Yu. A. Brychkov, and O. I. Marichev, Integrals and Series. Volume 1: Elementary Functions. Gordon and Breach Science Publishers / CRC Press. New York-London. 1986, 798 pages. 